\title{
Black hole nonmodal linear stability: the Schwarzschild (A)dS cases
}

\author{
Gustavo Dotti* \\ Facultad de Matemática, Astronomía y Física, \\ Universidad Nacional de Córdoba, \\ Instituto de Física Enrique Gaviola, Conicet. \\ Ciudad Universitaria, (5000) Córdoba, Argentina
}

\begin{abstract}
The nonmodal linear stability of the Schwarzschild black hole established in Phys. Rev. Lett. 112 (2014) 191101 is generalized to the case of a nonnegative cosmological constant $\Lambda$. Two gauge invariant combinations $G_{ \pm}$of perturbed scalars made out of the Weyl tensor and its first covariant derivative are found such that the map $\left[h_{\alpha \beta}\right] \rightarrow\left(G_{-}\left(\left[h_{\alpha \beta}\right]\right), G_{+}\left(\left[h_{\alpha \beta}\right]\right)\right)$ with domain the set of equivalent classes $\left[h_{\alpha \beta}\right]$ under gauge transformations of solutions of the linearized Einstein's equation, is invertible. The way to reconstruct a representative of $\left[h_{\alpha \beta}\right]$ in terms of $\left(G_{-}, G_{+}\right)$is given. It is proved that, for an arbitrary perturbation consistent with the background asymptote, $G_{+}$ and $G_{-}$are bounded in the the outer static region. At large times, the perturbation decays leaving a linearized Kerr black hole around the Schwarzschild or Schwarschild de Sitter background solution. For negative cosmological constant it is shown that there are choices of boundary conditions at the time-like boundary under which the Schwarzschild anti de Sitter black hole is unstable. The root of Chandrasekhar's duality relating odd and even modes is exhibited, and some technicalities related to this duality and omitted in the original proof of the $\Lambda=0$ case are explained in detail.
\end{abstract}

\section{CONTENTS}

I. Introduction

II. Tensor fields on a spherically symmetric space-time $\quad 6$

A. Covector and symmetric tensor fields $\quad 7$

B. The Schwarzschild (A)dS solution 9

1. Horizons and the static region $\quad 10$

2. The bifurcation sphere at $r=r_{h} \quad 10$

3. Hyperbolic equations and global hyperbolicity of the static region 11

III. The Linearized Einstein equation: odd sector 13

A. Gauge transformations and gauge invariants 13

B. Solution of the linearized Einstein equation 14

C. Measurable effects of the perturbation on the geometry 16

D. Nonmodal linear stability of the $\Lambda \geq 0$ black holes 18

1. The asymptotically flat case 18

2. The asymptotically de Sitter case 19

3. A comment on the asymptotically anti de Sitter case 19

IV. The Linearized Einstein equation: even sector $r$

A. Gauge transformations and gauge invariants 19

1. $\ell=0$ mode $\quad 20$

2. $\ell=1$ modes $\quad 20$

3. $\ell>1$ modes 21

B. The linearized Einstein equation 21

1. $\ell>1$ modes 21

2. $\ell=0$ mode $\quad 22$

3. $\ell=1$ modes 23

C. Solution of the linearized Einstein equation 23

D. The ubiquitous Regge-Wheeler equation 24

E. Chandrasekhar's duality 27

* gdotti@famaf.unc.edu.ar 
1. Case $\Lambda \geq 0$

2. Case $\Lambda<0$ : instability of SAdS 28

F. Measurable effects of the perturbation on the geometry 30

G. Non-modal linear stability of the $\Lambda \geq 0$ black holes 31

V. Discussion 34

A. Evolution of perturbations 34

B. Reduction of the LEE to the 4DRW equation 35

VI. Acknowledgments 35

References $\quad 36$

\section{INTRODUCTION}

One of the most salient open problems in classical General Relativity (GR) is proving the stability of the outer region of the stationary electro-vacuum black holes in the Kerr-Newman family. A complete proof of stability in the context of the non linear GR equations has only been given for Minkowski spacetime [6]; the stability problem of more complex solutions of Einstein's equation is usually approached by analyzing the behavior of linear test fields satisfying appropriate boundary conditions in order to establish if unbounded solutions are allowed. Scalar test fields provide a first clue, whereas gravitational waves, that is, metric perturbations $h_{\alpha \beta}$ propagating on the background spacetime $\left(\mathcal{M}, g_{\alpha \beta}\right)$, give a more realistic approach to the problem. For vacuum spacetimes, assuming a cosmological constant $\Lambda$, the metric perturbation $h_{\alpha \beta}$ satisfies the linearized Einstein equation (LEE)

$$
\mathcal{E}\left[h_{\alpha \beta}\right]:=-\frac{1}{2} \nabla^{\gamma} \nabla_{\gamma} h_{\alpha \beta}-\frac{1}{2} \nabla_{\alpha} \nabla_{\beta}\left(g^{\gamma \delta} h_{\gamma \delta}\right)+\nabla^{\gamma} \nabla_{(\alpha} h_{\beta) \gamma}-\Lambda h_{\alpha \beta}=0,
$$

obtained by assuming that on a fixed four dimensional manifold $\mathcal{M}$ (the spacetime) there is a mono-parametric family of solutions $g_{\alpha \beta}(\epsilon)$ of the vacuum Einstein field equation

$$
R_{\alpha \beta}[g(\epsilon)]-\Lambda g_{\alpha \beta}(\epsilon)=0
$$

around the "unperturbed background" $g_{\alpha \beta}=g_{\alpha \beta}(0)$, and Taylor expanding $(2)$ at $\epsilon=0$. For tensor fields that depend functionally on the metric we use a dot to denote the "perturbed field", which is the field obtained by taking the first derivative with respect to $\epsilon$ at $\epsilon=0$, e.g., for the Riemann tensor,

$$
\dot{R}_{\beta \gamma \delta}^{\alpha}=\left.\frac{d}{d \epsilon}\right|_{\epsilon=0} R_{\beta \gamma \delta}^{\alpha}[g(\epsilon)] .
$$

We make an exception for the metric field itself by adopting the standard notation $h_{\alpha \beta}=\dot{g}_{\alpha \beta}$ and, as usually done in linear perturbation theory, defining $h_{\beta}^{\alpha}=g^{\alpha \mu} h_{\mu \beta}$, and $h^{\alpha \beta}=g^{\alpha \mu} g^{\beta \nu} h_{\mu \nu}$. Note that this convention implies that

$$
\dot{g}^{\alpha \beta}=-h^{\alpha \beta} .
$$

Equation (1) is the first order Taylor coefficient of (2) around $\epsilon=0$, that is $\mathcal{E}\left[h_{\alpha \beta}\right]=\dot{R}_{\alpha \beta}-\Lambda h_{\alpha \beta}$. Trivial solutions of this equation are

$$
h_{\alpha \beta}=£_{\xi} g_{\alpha \beta}=\nabla_{\alpha} \xi_{\beta}+\nabla_{\beta} \xi_{\alpha}
$$

where $\xi^{\alpha}$ is an arbitrary vector field; these amount to the first order in $\epsilon$ change of the metric under the pullback by the flow $\Phi_{\epsilon}^{\xi}: \mathcal{M} \rightarrow \mathcal{M}$ generated by the vector field $\xi^{\alpha}$. Any two solutions $h_{\alpha \beta}$ and $h_{\alpha \beta}^{\prime}$ such that

$$
h_{\alpha \beta}^{\prime}=h_{\alpha \beta}+£_{\xi} g_{\alpha \beta}
$$

are related by this diffeomorphism and thus physically equivalent, this being the gauge freedom of linearized gravity. If a tensor field $T$ is a functional of the metric $g$, then $\dot{T}$ is a linear functional of $h_{\alpha \beta}$ and, under the gauge transformation (5) we find that

$$
\dot{T}\left[h^{\prime}\right]=\dot{T}[h]+£_{\xi} T .
$$


In particular, if $T$ is a scalar field, $\dot{T}$ is gauge invariant iff $0=£_{\xi} T=\xi^{\alpha} \partial_{\alpha} T$ for every vector field, that is, if $T$ is constant in the background.

Curvature related scalar fields (CS for short, not to be confused with the tetrad components of the Weyl tensor in the Newman-Penrose formalism), are scalar fields obtained by a full contraction of a polynomial in the Riemann tensor and its covariant derivatives, the metric and the volume form. Although these fields partially characterize the metric, it is well known that the information they carry is limited, an extreme example of this fact being the vanishing scalar invariants spacetimes, which have a nonzero Riemann tensor and yet every CS vanishes [24]. This fact may suggest that the perturbation of CS under a given solution $h_{\alpha \beta}$ of the LEE provide very limited information on $h_{\alpha \beta}$. It was shown in [9], however, that for a Schwarzschild black hole background, there are two gauge invariant combinations $G_{ \pm}$of perturbed CSs that fully characterize the gauge equivalence class $\left[h_{\alpha \beta}\right]$ of the corresponding solution of the LEE. More precisely, let $C^{\alpha}{ }_{\beta \gamma \delta}$ be the Weyl tensor, $\varepsilon_{\alpha \beta \mu \nu}$ the volume form, and consider the CSs

$$
Q_{-}=\frac{1}{96} C^{\alpha \beta \gamma \delta} \varepsilon_{\alpha \beta \mu \nu} C_{\gamma \delta}^{\mu \nu}, \quad Q_{+}=\frac{1}{48} C^{\alpha \beta \gamma \delta} C_{\alpha \beta \gamma \delta}, \quad X=\frac{1}{720}\left(\nabla_{\epsilon} C_{\alpha \beta \gamma \delta}\right)\left(\nabla^{\epsilon} C^{\alpha \beta \gamma \delta}\right) .
$$

The background values of these fields, that is, their value for the $\Lambda=0$ Schwarzschild solution, are $Q_{-}^{0}{ }_{\text {Schw }}=0$, $\underset{+S c h w}{0}=M^{2} / r^{6}$ and $X_{S c h w}^{0}=M^{2}(r-2 M) / r^{9}$, where $M$ is the mass and $r$ the areal radius, then the fields

$$
G_{-}=\dot{Q}_{-}, \quad G_{+}=(9 M-4 r) \dot{Q}_{+}+3 r^{3} \dot{X},
$$

made out of the first order variations of these CS, are gauge invariant. It was shown in [9] that the linear map

$$
\left[h_{\alpha \beta}\right] \rightarrow\left(G_{-}\left(\left[h_{\alpha \beta}\right]\right), G_{+}\left(\left[h_{\alpha \beta}\right]\right)\right)
$$

with domain the equivalence classes of smooth solutions of the LEE preserving the asymptotic flatness, is injective. This implies that the scalars $G_{ \pm}$, beyond measuring the "amount of distortion" caused by the perturbation, encode all the relevant information on the perturbation class $\left[h_{\alpha \beta}\right]$. A way to construct a class representative $h_{\alpha \beta}$ from the fields $G_{ \pm}\left(\left[h_{\alpha \beta}\right]\right)$ is indicated in [9].

The stability concept introduced in [9] is based on i) the existence of the CSs $G_{ \pm}$for which (9) is injective on smooth solutions of the LEE that preserve the asymptotic flatness, and ii) the proof that for this class of solutions of the LEE the scalar fields $G_{ \pm}\left(\left[h_{\alpha \beta}\right]\right)$ are bounded. More precisely, it is proved in [9] that in the outer region $r \geq 2 M$

$$
\left|G_{-}\right|<\frac{K_{-}}{r^{6}}, \quad\left|G_{+}\right|<\frac{K_{+}}{r^{3}},
$$

where $K_{ \pm}$are constants that can be obtained from the perturbation field datum at a Cauchy slice. Given that the scalars $G_{ \pm}$contain all the gauge invariant information on the perturbation, the fact that they remain bounded as the perturbation evolves through spacetime gives a meaningful notion of linear stability.

This concept of stability, that we call nonmodal, should be compared with prior linear gravity stability notions for the Schwarzschild black hole. To this end we review the results in a short list of papers that were crucial in the development of this subject. It is important to stress that they all use the spherical symmetry of the Schwarzschild spacetime to decompose a metric perturbation

$$
h_{\alpha \beta}=\sum_{(\ell, m, p= \pm)} h_{\alpha \beta}^{(\ell, m, p= \pm)}
$$

into even $(p=+)$ and odd $(p=-)(\ell, m)$ modes. Here $\ell$ refers to the eigenspace of the Laplace-Beltrami operator acting on real scalar fields on $S^{2}$ corresponding to the eigenvalue $-\ell(\ell+1), m$ is an index labeling a particular basis of this $2 \ell+1$ dimensional space, and the parity $p$ accounts for the way $h_{\alpha \beta}^{(\ell, m, p= \pm)}$ transforms when pull-backed by the antipodal map on $S^{2}$ (for details refer to Section II A).

The first work on the linear stability of the Schwarzschild black hole is T. Regge and J. Wheeler 1957 paper [26], where the decomposition (11) was proposed and the $\ell=0,1$ modes where recognized to be non-dynamical. At the time the very notion of black hole was unclear (the term "black hole" was coined by J. Wheeler some ten years later), and Kerr's solution had not yet been discovered. This is probably why, although the $\ell=0$ even piece of the perturbation was readily identified as a mass shift in [26], the odd $\ell=1$ modes, which corresponds to perturbing along a Kerr family $g_{\alpha \beta}(\epsilon)$ with $\epsilon=J / M$, was misunderstood (see the paragraph between equations (37) and (38) in [26]) and the opportunity of producing a "slowly rotating" black hole at a time when there was no clue about a rotating black hole solution was missed [? ]. The decomposition (11) in [26] was done in Schwarzschild coordinates $(t, r, \theta, \phi)$ in 
what came to be called the Regge-Wheeler (RW, for short) gauge. The LEE in the even and odd sectors were shown to decouple, and the dynamical odd sector of the LEE reduced to an infinite set of two dimensional wave equations, individually know as the RW equation:

$$
\left(\partial_{t}^{2}-\partial_{r^{*}}^{2}+f V_{(\ell, m)}^{R W}\right) \phi_{(\ell, m)}^{-}=0, \quad \ell \geq 2,
$$

where (adding a cosmological constant $\Lambda$ for future reference) $\partial_{r^{*}}=f \partial_{r}, f=1-2 M / r-\Lambda r^{2} / 3$, and

$$
V_{(\ell, m)}^{R W}=\frac{\ell(\ell+1)}{r^{2}}-\frac{6 M}{r^{3}}
$$

The $\ell \geq 2$ even sector LEE, a much more intricate system of equations, was disentangled by F. Zerilli in his 1970 paper [32] and shown to be equivalent to the wave equations

$$
\left(\partial_{t}^{2}-\partial_{r^{*}}^{2}+f V_{(\ell, m)}^{Z}\right) \phi_{(\ell, m)}^{+}=0, \quad \ell \geq 2
$$

with potentials

$$
V_{(\ell, m)}^{Z}=\frac{\left[\mu^{2} \ell(\ell+1)-24 M^{2} \Lambda\right] r^{3}+6 \mu^{2} M r^{2}+36 \mu M^{2} r+72 M^{3}}{r^{3}(6 M+\mu r)^{2}}, \quad \mu=(\ell-1)(\ell+2) .
$$

For $\Lambda \neq 0$ the RW and Zerilli potentials first appeared in [17]. The solution of the LEE in the RW gauge is then given in the form

$$
{ }^{(R W)} h_{\alpha \beta}^{(\ell, m, p= \pm)}=\mathcal{D}_{\alpha \beta}^{(\ell, m, p= \pm)}\left[\phi_{(\ell, m)}^{ \pm}, S_{(\ell, m)}\right]
$$

where $\mathcal{D}_{\alpha \beta}^{(\ell, m, p= \pm)}$ is a bilinear differential operator acting on $\phi_{(\ell, m)}^{ \pm}$and the spherical harmonics $S_{(\ell, m)}$. Note that, since focus is on non-stationary modes, only $\ell \geq 2$ enter this formulation. The non-stationary solution space of the LEE is thus parametrized by the infinite set of scalar fields $\phi_{(\ell, m)}^{ \pm}$that enter the series $(11)$ through (14).

Every notion of gravitational linear stability of the Schwarzschild black hole prior to [9] was concerned with the behavior of the potentials $\phi_{(\ell, m)}^{ \pm}$of isolated $(\ell, m)$ modes (we call this "modal linear stability"). In particular:

- In [26] it was shown that separable solutions $\phi_{(\ell, m)}^{-}=\Re e^{i \omega t} \chi_{(\ell, m)}^{-}(r)$ that do not diverge as $r \rightarrow \infty$ require $\omega \in \mathbb{R}$, ruling out exponentially growing solutions in the odd sector.

- In [32] it was shown that separable solutions $\phi_{(\ell, m)}^{+}=\Re e^{i \omega t} \chi_{(\ell, m)}^{+}(r)$ that do not diverge as $r \rightarrow \infty$ require $\omega \in \mathbb{R}$, ruling out exponentially growing solutions in the even sector.

- In [25] it was shown that, for large $t$ and fixed $r, \phi_{(\ell, m)}^{ \pm}(t, r)$ decays as $t^{-(2 \ell+2)}$ (an effect known as "Price tails").

- In [29], the conserved energy

$$
\int_{2 M}^{\infty}\left[\left(\partial_{t} \phi_{(\ell, m)}^{ \pm}\right)^{2}+\left(\partial_{r^{*}} \phi_{(\ell, m)}^{ \pm}\right)^{2}+\phi_{(\ell, m)}^{ \pm} f V_{(\ell, m)}^{R W / Z} \phi_{(\ell, m)}^{ \pm}\right] f d r
$$

was used to rule out uniform exponential growth in time.

- Also in [29], a point wise bound on the RW and Zerilli potentials was found in the form

$$
\left|\phi_{(\ell, m)}^{ \pm}(t, r)\right|<C_{(\ell, m)}^{ \pm}, \quad r>2 M, \text { all } t
$$

where the constants $C_{(\ell, m)}^{ \pm}$are given in terms of the initial data $\left(\phi_{(\ell, m)}^{ \pm}\left(t_{o}, r\right), \partial_{t} \phi_{(\ell, m)}^{ \pm}\left(t_{o}, r\right)\right)$

To understand the limitations of these results it is important to keep in mind that the $\phi_{(\ell, m)}^{ \pm}$are an infinite set of potentials defined on the $(t, r)$ orbit space $\mathcal{M} / S O(3)$, whose first and second order derivatives $\phi_{(\ell, m)}^{ \pm}$enter the terms in the series (11) through (14), together with first and second derivatives of the spherical harmonics. Two extra derivatives are required to calculate the perturbed Riemann tensor, as a first step to measure the effects of the perturbation on the curvature. Thus, the relation of the potentials $\phi_{(\ell, m)}^{ \pm}$to geometrically meaningful quantities is 
remote, and the usefulness of the bounds (16) to measure the strength of the perturbation is far from obvious.

The motivation of the nonmodal approach can be better understood if we put in perspective the progress made in [29], equation (16). The fact that the two dimensional wave equations (RWE) and (ZE) can be solved by separating variables $\left(\phi_{(\ell, m)}^{ \pm}=e^{i \omega t} \psi(r)\right)$ had previous works on linear stability focus on showing that $\omega$ must be real, a limited notion of stability that does not even rule out, e.g., linear $t$ growth [29]. To obtain the type of bounds (16) one must "undo" the separation of variables and reconsider the original equations (RWE) and (ZE), as this allows to work out results that are valid for generic, non separable solutions. The idea behind the non modal stability concept introduced in this paper, is to go one step further and "undo" the separation of the $(\theta, \phi)$ variables that antecedes the reduction of the LEE to (RWE) and (ZE), in order to get bounds for truly four dimensional quantities. When trying to make this idea more precise, one is immediately faced to the problem of which four dimensional quantity one should look at. If this quantity is to measure the strength of the perturbation, it should be a field related to the curvature change, and it should also be a scalar field, since there is no natural norm for a tensor field in a Lorentzian background. If this scalar field does not obey a four dimensional wave equation (or some partial differential equation), we do not have a clear tool to place bounds on it. Thus, we are naturally led to the question of the existence of scalar fields made from perturbed CSs that, as a consequence of the LEE, obey some wave-like equation. This is the problem we address in this paper. The name nonmodal stability is borrowed from fluid mechanics, where the limitations of the normal mode analysis were realized some thirty years ago in experiments involving wall bounded shear flows [30]. In that problem, the linearized Navier-Stokes operator is non normal, so their eigenfunctions are non orthogonal and, as a consequence, even if they individually decay as $e^{i w t}, \Im(w)>0$, a condition that assures large $t$ stability, there may be important transient growths [30]. Take, e.g., the following simple example (from [30], section 2.3 and figure 2) of a system of two degrees of freedoms: $\vec{v} \in \mathbb{R}^{2}$ obeying the equation $d \vec{v} / d t=-A \vec{v}$, with $\left[A, A^{T}\right] \neq 0$ a matrix with $($ non orthogonal!) eigenvectors $\vec{e}_{1}, \vec{e}_{2}$, say,

$$
A=\left(\begin{array}{ll}
1 & \gamma \\
0 & 2
\end{array}\right), \quad \vec{e}_{1}=\left(\begin{array}{l}
1 \\
0
\end{array}\right), \quad \vec{e}_{2}=\left(\begin{array}{c}
1 \\
\gamma^{-1}
\end{array}\right)
$$

Consider the case $\gamma>>1$. Note that, although $\omega_{1}=i, \omega_{2}=2 i$, that is, normal modes decay exponentially, if $\vec{v}(0)=\alpha\left(\vec{e}_{1}-\vec{e}_{2}\right)$, then $\vec{v}(t)=\alpha \vec{e}_{1} e^{-t}-\alpha \vec{e}_{2} e^{-2 t}$ reaches a maximum norm $\|\vec{v}\| \simeq(\gamma / 4)\|\vec{v}(0)\|$ at a finite time before decaying to zero.

For the odd sector of the LEE, a four dimensional approach relating the metric perturbation with a scalar potential $\Phi$ defined on $\mathcal{M}$ (instead of $\mathcal{M} / S O(3))$ was found in [9], where it was noticed that the sum over $(\ell, m)$ of $(14)$ simplifies to

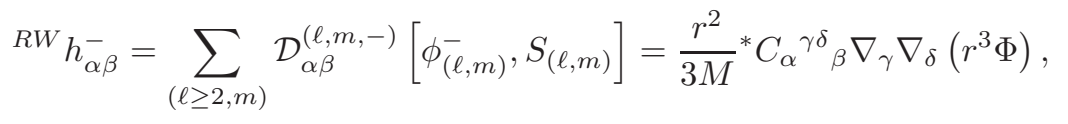

where $\Phi$ is a field assembled using spherical harmonics and the RW potentials:

$$
\Phi=\sum_{(\ell \geq 2, m)} \frac{\phi_{(\ell, m)}^{-}}{r} S_{(\ell, m)}: \mathcal{M} \rightarrow \mathbb{R}
$$

The odd sector LEE equations (RWE) for $\phi_{(\ell, m)}^{-}: \mathcal{M} / S O(3) \rightarrow \mathbb{R}$ combined to the spherical harmonic equations for the spherical harmonics $S_{(\ell, m)}: S^{2} \rightarrow \mathbb{R}$, turn out to be equivalent to what we call the four dimensional Regge-Wheeler equation which, adding a cosmological constant, reads

$$
\nabla^{\alpha} \nabla_{\alpha} \Phi+\left(\frac{8 M}{r^{3}}-\frac{2 \Lambda}{3}\right) \Phi=0
$$

Note however that $\Phi$ is no more than the collection of $\phi_{(\ell, m)}^{-}$'s, so its connection to geometrically relevant fields is loose. Much more important is the fact, also proved in [9] for $\Lambda=0$, that the LEE implies that the field $r^{5} \dot{Q}_{-}=r^{5} G_{-}$ also satisfies the 4DRWE, as this is what allows us to place a point wise bound on $G_{-}=\dot{Q}_{-}$.

The even sector of the LEE is more difficult to approach. Is the simplicity of the RW potential (12), with the obvious $\ell(\ell+1) / r^{2}$ term, what suggested considering the field (19). The way $\ell$ appears in (13), instead, is a clear indication that there is no natural 4D interpretation of (ZE). Is a map exchanging solutions of the RWE and ZE equations, found by Chandrasekhar [4], what ultimately allows us to also reduce the even non stationary LEE equations to (4DRWE). 
As a consequence, the entire set of non stationary LEE reduces to two fields satisfying equation (4DRWE), as stated in Theorem 4 below.

The purpose of this paper is twofold: (i) to extend the results in [9] to Schwarzschild black holes in cosmological backgrounds, and (ii) to explain in detail a number of technicalities omitted in [9] due to the space limitations imposed by the letter format. For $\Lambda \geq 0$ we give a proof of non-modal stability. We leave aside the treatment of stability of the Schwarzschild anti de Sitter (SAdS) black hole, since the issue of non global hiperbolicity and ambiguous dynamics due to the conformal timelike boundary takes us away from the core of the subjects addressed here. We show however that there is (at least) one choice of Robin boundary condition at the time-like boundary for which there is an instability, and we explicitly exhibit this instability and its effect on the background geometry. To the best of our knowledge, this has not been informed before. A systematic study of the gravitational linear stability of SAdS black holes under different boundary conditions is to be found in [33].

We have found that $\left(G_{+}, G_{-}\right)$defined in (8) are appropriate variables to study the most general gravitational linear perturbations of Schwarzschild (A)dS black holes. The $\ell=0,1$ pieces of these fields encode the relevant information on the stationary modes, which are perturbations within the Kerr family (parametrized by mass $M$ and the angular momenta components), whereas the $\ell \geq 2$ terms encode the dynamics. More precisely:

- $G_{-}$contains no $\ell=0$ term, time independent $\ell=1$ terms proportional to the first order angular momenta components $j^{(i)}$, and a time dependent $\ell>1$ piece obeying the 4DRW equation.

- $G_{+}$contains no $\ell=1$ term, a time independent $\ell=0$ piece proportional to a mass shift $\dot{M}$, and a time dependent $\ell>1$ piece which, for $\Lambda \geq 0$, can be written in terms of fields obeying the 4DRW equation.

Once the appropriate set of perturbation fields $\left(G_{+}, G_{-}\right)$is given, and their relation to the 4DRW equation established for $\Lambda \geq 0$, we may adapt to the $4 \mathrm{DRW}$ equation the techniques used to prove boundedness of solutions of the scalar wave equation, in order to analyze the behavior of the $G_{ \pm}$fields. As an example, the result of Kay and Wald [21] was used in [9] to prove (10) in the Schwarzschild case, and is adapted here to prove that (10) holds also for positive $\Lambda$. We can go further and take advantage of the growing literature on decay of solutions of the scalar wave equation on S(A)dS backgrounds, as many of these results are expected to hold also for (4DRWE). Specific time decay results for the 4DRW equation, somewhat expected from Price's result [25], can be found in [1] (see also the recent preprint $[7])$. Putting together the bijection (9), the above description of the stationary $(\ell=0,1)$ and dynamic $(\ell \geq 2)$ pieces of $G_{ \pm}$, and the time decay results, the following picture emerges for a perturbed Schwarzschild (SdS) black hole: a generic perturbation contains a mass shift, infinitesimal angular momenta $j^{(i)}$ and dynamical degrees of freedom; at large times the dynamical degrees of freedom decay and what is left is a linearized Kerr (Kerr dS) black hole around the background Schwarzschild (SdS) solution.

Through the paper, calculations are carried leaving $\Lambda$ unspecified whenever possible, and specializing when necessary. Among the many current treatments of linear perturbations of spherically symmetric spacetimes, we have made heavy use of the excellent paper [5], which we found particularly well suited to our approach.

\section{TENSOR FIELDS ON A SPHERICALLY SYMMETRIC SPACE-TIME}

A spherically symmetric space-time is a warped product $\mathcal{M}=\mathcal{O} \times_{r^{2}} S^{2}$ of a Lorentzian two-manifold $(\mathcal{O}, \tilde{g})$ with the unit sphere $\left(S^{2}, \widehat{g}\right)$, for which we will use the standard angular coordinates $\widehat{g}_{A B} d y^{A} d y^{B}=d \theta^{2}+\sin ^{2} \theta d \phi^{2}$ :

$$
g_{\alpha \beta} d z^{\alpha} d z^{\beta}=\tilde{g}_{a b}(x) d x^{a} d x^{b}+r^{2}(x) \widehat{g}_{A B}(y) d y^{A} d y^{B} .
$$

The form of the metric (20) implies that $(\mathcal{M}, g)$ inherits the isometry group $O(3)=S O(3) \times P$ of $\left(S^{2}, \widehat{g}\right)$ as an isometry subgroup. Here $S O(3)$ are the proper rotations and $P$ is the antipodal map $P(\theta, \phi)=(\pi-\theta, \phi+\pi)$. Since $S O(3)$ acts transitively on $S^{2}$, we find that $\mathcal{O}=\mathcal{M} / S O(3)$, this is why $(\mathcal{O}, \tilde{g})$ is called the $(S O(3))$ orbit space.

Equation (20) exhibits our index conventions, which we have adopted form ref [5]: lower case Latin indexes are used for tensors on $\mathcal{O}$, upper case Latin indexes for tensors on $S^{2}$, and Greek indexes for space-time tensors. We will furthermore assume that

$$
\alpha=(a, A), \beta=(b, B), \gamma=(c, C), \delta=(d, D), \ldots
$$

Tensor fields introduced with a lower $S^{2}$ index (say $Z_{A}$ ) and then shown with an upper $S^{2}$ index are assumed to have been acted upon with the unit $S^{2}$ metric inverse $\widehat{g}^{A B}$, (i.e., $Z^{A} \equiv \widehat{g}^{A B} Z_{B}$ ), and similarly for upper $S^{2}$ indexes 
moving down. $\widetilde{D}_{a}, \tilde{\epsilon}_{a b}$ and $\tilde{g}^{a b}$ are the covariant derivative, volume form (any chosen orientation) and metric inverse for $(\mathcal{O}, \tilde{g}) ; \widehat{D}_{A}$ and $\widehat{\epsilon}_{A B}$ are the covariant derivative and volume form on the unit sphere, for which we assume the standard orientation $\widehat{\epsilon}=\sin (\theta) d \theta \wedge d \phi$.

As an example, in terms of the differential operators $\widetilde{D}_{a}$ and $\widehat{D}_{A}$, the Laplacian on scalar fields reads

$$
\nabla_{\alpha} \nabla^{\alpha} \Phi=\widetilde{D}_{a} \widetilde{D}^{a} \Phi+\frac{2}{r}\left(\widetilde{D}^{b} r\right)\left(\widetilde{D}_{b} \Phi\right)+\frac{1}{r^{2}} \widehat{D}_{A} \widehat{D}^{A} \Phi
$$

\section{A. Covector and symmetric tensor fields}

The Einstein field equation, as well as its linearized version around a particular solution, is expressed as an equality among symmetric tensor fields. The metric perturbation $h_{\alpha \beta}$ may be subjected to gauge transformations of the form (5). This is why we are interested in the decomposition on a spherically symmetric spacetime of covector fields (such as $\left.\zeta_{\alpha}\right)$ and symmetric rank two tensor fields such as $h_{\alpha \beta}$.

We will assume all tensor fields on $\mathcal{M}$ are smooth. As a consequence their components will be square integrable on $S^{2}$ and can be expanded using a real orthonormal basis of scalar spherical harmonics

$$
\begin{gathered}
\widehat{D}^{A} \widehat{D}_{A} S_{(\ell, m)}+\ell(\ell+1) S_{(\ell, m)}=0, \\
\int_{S^{2}} S_{\left(\ell^{\prime}, m\right)} S_{(\ell, m)} \widehat{\epsilon}=\delta_{\ell \ell^{\prime}} \delta_{m m^{\prime}},
\end{gathered}
$$

where $m$ numbers an orthonormal basis $S_{(\ell, m)}$ of the $(2 \ell+1)$-dimensional eigenspace with eigenvalue $-\ell(\ell+1)$ of the Laplace-Beltrami operator $\widehat{D}^{A} \widehat{D}_{A}$ on scalar functions $(\ell=0,1,2,3, \ldots)$. An explicit choice for the $\ell=0,1$ subspaces is $S_{(0,0)}=\sqrt{\frac{1}{4 \pi}}$, and

$$
\begin{aligned}
& S_{(1,1)}=\sqrt{\frac{3}{4 \pi}} \sin \theta \cos \phi \\
& S_{(1,2)}=\sqrt{\frac{3}{4 \pi}} \sin \theta \sin \phi \\
& S_{(1,3)}=\sqrt{\frac{3}{4 \pi}} \cos \theta .
\end{aligned}
$$

We denote $L^{2}\left(S^{2}\right)_{\ell}$ the $\ell$ subspace of $L^{2}\left(S^{2}\right)$ and $L^{2}\left(S^{2}\right)_{>j}=\bigoplus_{\ell>j} L^{2}\left(S^{2}\right)_{\ell}$.

A covector field on $\mathcal{M}$

$$
\xi_{\alpha}=\left(\xi_{a}, \xi_{A}\right)
$$

contains the $S^{2}$ covector $\xi_{A}$ which, according to Proposition 2.1 in [20], can be uniquely decomposed as $\xi_{A}=\widehat{D}_{A} a+V_{A}$ where $\widehat{D}^{A} V_{A}=0$. This last condition implies that $V_{A}$ is dual to the differential of an $S^{2}$-scalar, $V_{A}=\widehat{\epsilon}_{A}{ }^{C} \widehat{D}_{C} b$. It then follows that, introducing $a=r^{2} X$ and $b=r^{2} Y$ for later convenience,

$$
\xi_{\alpha}=\xi_{\alpha}^{(-)}+\xi_{\alpha}^{(+)},
$$

where the odd piece of $\xi_{\alpha}$ is

$$
\xi_{\alpha}^{(-)}=\left(0, r^{2} \widehat{\epsilon}_{A}^{C} \widehat{D}_{C} Y\right)
$$

and its even piece is

$$
\xi_{\alpha}^{(+)}=\left(\xi_{a}, r^{2} \widehat{D}_{A} X\right) .
$$

For a given covector field $\xi_{\alpha}$, the scalar fields $X, Y: \mathcal{M} \rightarrow \mathbb{R}$ are unique up to an $S^{2}$-constant, thus they are unique if we require that they belong to $L^{2}\left(S^{2}\right)>0$

$$
\int_{S^{2}} X \widehat{\epsilon}=0=\int_{S^{2}} Y \widehat{\epsilon}
$$


a condition that we will assume. The symmetric tensor field $h_{\alpha \beta}=h_{\beta \alpha}$

$$
h_{\alpha \beta}=\left(\begin{array}{ll}
h_{a b} & h_{a B} \\
h_{A b} & h_{A B}
\end{array}\right)
$$

contains two $S^{2}$ covector fields

$$
h_{a B}=\widehat{D}_{B} q_{a}+\widehat{\epsilon}_{B}^{C} \widehat{D}_{C} h_{a}
$$

and a symmetric $S^{2}$ tensor field $h_{A B}$. Note that $q_{a}$ and $h_{a}$ are covector fields on $\mathcal{O}$ parametrized on $S^{2}$.

Using Proposition 2.2 in [20] and the fact that there are no transverse traceless symmetric tensor fields on $S^{2}$ [18], we find that the $S^{2}$ symmetric tensor $h_{A B}$ in (31) can be uniquely decomposed into three terms:

$$
h_{A B}=2 \widehat{D}_{(A}\left(\widehat{\epsilon}_{B) C} \widehat{D}^{C} L\right)+\left(2 \widehat{D}_{A} \widehat{D}_{B}-\widehat{g}_{A B} \widehat{D}^{C} \widehat{D}_{C}\right) S_{1}+\widehat{g}_{A B} \frac{S_{2}}{2}
$$

Introducing $J=S_{2} / r^{2}, G=S_{1} / r^{2}$ and $k=L / r^{2}$ we arrive at (c.f., [5], Section IV.A)

Lemma 1. A generic smooth metric perturbation admits the following decomposition:

$$
h_{\alpha \beta}=h_{\alpha \beta}^{(-)}+h_{\alpha \beta}^{(+)}
$$

where the odd piece of $h_{\alpha \beta}$ is

$$
h_{\alpha \beta}^{(-)}=\left(\begin{array}{cc}
0 & \widehat{\epsilon}_{B}{ }^{C} \widehat{D}_{C} h_{a} \\
\widehat{\epsilon}_{A}{ }^{C} \widehat{D}_{C} h_{b} & 2 r^{2} \widehat{\epsilon}_{(A}{ }^{C} \widehat{D}_{B)} \widehat{D}_{C} k
\end{array}\right)
$$

and the even piece is

$$
h_{\alpha \beta}^{(+)}=\left(\begin{array}{cc}
h_{a b} & \widehat{D}_{B} q_{a} \\
\widehat{D}_{A} q_{b} & r^{2}\left[\frac{J}{2} \widehat{g}_{A B}+\left(2 \widehat{D}_{A} \widehat{D}_{B}-\widehat{g}_{A B} \widehat{D}^{C} \widehat{D}_{C}\right) G\right]
\end{array}\right) .
$$

The proof of the following lemma follows from straightforward calculations:

\section{Lemma 2.}

(i) The kernel of the map $\left(h_{a}, k\right) \rightarrow h_{\alpha \beta}^{(-)}$defined in (35) is the set of $h_{a}$ and $k$ of the form

$$
h_{a}=h_{a}(x), \quad k=k_{1}(x)+k_{2}(x) \cos (\theta)+\sin (\theta)\left(k_{3}(x) \cos (\phi)+k_{4}(x) \sin (\phi)\right),
$$

This implies that $h_{a}$ and $k$ are unique if they are required to belong to $L^{2}\left(S^{2}\right)_{>0}$ and $L^{2}\left(S^{2}\right)_{>1}$ respectively:

$$
h_{a}=\sum_{\ell \geq 1, m} h_{a}^{(\ell, m)}(x) S_{(\ell, m)}(\theta, \phi), \quad k(x)=\sum_{\ell \geq 2, m} k^{(\ell, m)}(x) S_{(\ell, m)}(\theta, \phi) .
$$

(ii) The kernel of the map $\left(h_{a b}, q_{a}, J, G\right) \rightarrow h_{\alpha \beta}^{(+)}$defined in (36) is characterized by $h_{a b}=0, J=0$,

$$
q_{a}=q_{a}(x), \quad G=G_{1}(x)+G_{2}(x) \cos (\theta)+\sin (\theta)\left(G_{3}(x) \cos (\phi)+G_{4}(x) \sin (\phi)\right),
$$

thus, the fields $\left(h_{a b}, q_{a}, J, G\right)$ are uniquely defined if we require that $q_{a} \in L^{2}\left(S^{2}\right)_{>0}$ and $G \in L^{2}\left(S^{2}\right)_{>1}$.

From now on we will assume the required conditions for uniqueness of $k, J, G, h_{a}, q_{a}$ and $h_{a b}$.

The linearized Ricci tensor $\dot{R}_{\alpha \beta}$ admits a decomposition analogous to (31)-(36). Given that $S^{2}$-scalar fields, divergence free covector fields (which are all of the form $\widehat{\epsilon}_{A}{ }^{B} \widehat{D}_{B} C$ ) and transverse traceless symmetric tensors on $S^{2}$ span inequivalent $O(3)$ representations, and that the linear map $h_{\alpha \beta} \rightarrow \dot{R}_{\alpha \beta}$ is $O(3)$ invariant, this map cannot mix odd and even sectors [20]. This implies that $\dot{R}_{\alpha \beta}^{(+)}$is a linear functional of $h_{\alpha \beta}^{(+)}$only, and similarly $\dot{R}_{\alpha \beta}^{(-)}$depends only on $h_{\alpha \beta}^{(-)}$. 
Note from (25) that

$$
J_{(m)}^{A}=\sqrt{\frac{4 \pi}{3}} \widehat{\epsilon}^{A B} \widehat{D}_{B} S_{(1, m)},
$$

$m=1,2,3$, is a basis of Killing vector fields on $S^{2}$ generating rotations around orthogonal axis, normalized such that the maximum length of their orbits is $2 \pi$. The square angular momentum operator is the sum of the squares of the Lie derivatives along these Killing vector fields:

$$
\mathbf{J}^{2}=\sum_{m=1}^{3}\left(£_{J_{(m)}}\right)^{2} .
$$

This operator commutes with the maps $\left(h_{a}, k\right) \rightarrow h_{\alpha \beta}^{(-)}$and $h_{\alpha \beta}^{(-)} \rightarrow R_{\alpha \beta}^{(-)}$(and similarly in the even sector). As a consequence, the $(\ell, m)$ piece of $R_{\alpha \beta}^{(-)}$depends only on the $(\ell, m)$ piece of $h_{\alpha \beta}^{(-)}$(see equation (38)):

$$
h_{\alpha \beta}^{(\ell, m,-)}=\left(\begin{array}{cc}
0 & h_{a}^{(\ell, m)} \widehat{\epsilon}_{B}{ }^{C} \widehat{D}_{C} S_{(\ell, m)} \\
h_{b}^{(\ell, m)} \widehat{\epsilon}_{A}{ }^{C} \widehat{D}_{C} S_{(\ell, m)} & 2 r^{2} k^{(\ell, m)} \widehat{\epsilon}_{(A}{ }^{C} \widehat{D}_{B)} \widehat{D}_{C} S_{(\ell, m)}
\end{array}\right),
$$

and similarly in the even sector. Note from (42) and (40) that the odd $\ell=1$ modes add up to

$$
h_{\alpha \beta}^{(\ell=1,-)}=\left(\begin{array}{cc}
0 & \sum_{m=1}^{3} \sqrt{\frac{3}{4 \pi}} h_{a}^{(\ell=1, m)} J_{(m) B} \\
\sum_{m=1}^{3} \sqrt{\frac{3}{4 \pi}} h_{b}^{(\ell=1, m)} J_{(m) A} & 0
\end{array}\right) .
$$

These $\ell=1$ perturbations correspond to infinitesimal rotation, i.e., to deformations towards a stationary Kerr solution.

The $(\ell, m,+)$ mode is defined in a way analogous to (42), i.e., keeping a single term in the spherical harmonic expansion of the even fields $\left(h_{a b}, q_{a}, J, G\right)$. It is important to note that

$$
\begin{aligned}
& \mathbf{J}^{2} h_{\alpha \beta}^{(\ell, m, \pm)}=-\ell(\ell+1) h_{\alpha \beta}^{(\ell, m, \pm)}, \\
& P_{*} h_{\alpha \beta}^{(\ell, m, \pm)}= \pm(-1)^{\ell} h_{\alpha \beta}^{(\ell, m, \pm)} .
\end{aligned}
$$

The different behavior under parity is the signature that distinguishes odd from even modes.

\section{B. The Schwarzschild (A)dS solution}

The Schwarzschild / Schwarzschild (anti) de-Sitter $(\mathrm{S}(\mathrm{A}) \mathrm{dS}$ ) is the only spherically symmetric solution of the vacuum Einstein equation with a cosmological constant $\Lambda$ :

$$
R_{\alpha \beta}=\Lambda g_{\alpha \beta},
$$

$R_{\alpha \beta}$ the Ricci tensor of the Lorentzian metric $g_{\alpha \beta}$. The manifold is $\mathbb{R}_{v} \times(0, \infty)_{r} \times S^{2}$, the metric (c.f. equation (20))

$$
d s^{2}=-f d v^{2}+2 d v d r+r^{2}\left(d \theta^{2}+\sin ^{2} \theta d \phi^{2}\right), \quad f=1-\frac{2 M}{r}-\frac{\Lambda r^{2}}{3} .
$$

The constant $M$ is the mass of the solution, $M=0$ in (47) gives the Minkowski $(\Lambda=0)$, de Sitter $(\Lambda>0)$ and anti de Sitter $(\Lambda<0)$ spacetimes. $M>0$ in (47) corresponds to a Schwarzschild black hole if $\Lambda=0$, a Schwarzschild de Sitter (SdS) black hole if $\Lambda>0$ and $\Lambda<(3 M)^{-2}$, and SAdS black hole if $\Lambda<0$.

The Killing vector field $k^{a} \partial_{a}=\partial / \partial_{v}$ is timelike in the open sets defined by $f>0$ and spacelike in the open sets defined by $f<0$. The null hypersurfaces of constant positive $r$ where $f=0$ are the horizons, they cover the curvature singularity at $r=0$.

In any open set where $f \neq 0$ we may define a "tortoise" radial coordinate $r^{*}$ by

$$
\frac{d r^{*}}{d r}=\frac{1}{f}
$$


and a coordinate $t$ through

$$
t=v-r^{*}
$$

The metric in static coordinates $(t, r, \theta, \phi)$ is

$$
d s^{2}=-f d t^{2}+\frac{d r^{2}}{f}+r^{2}\left(d \theta^{2}+\sin ^{2} \theta d \phi^{2}\right)
$$

\section{Horizons and the static region}

The Schwarzschild $(\Lambda=0)$ and the SAdS $(\Lambda<0)$ black holes have a single horizon at $r=r_{h}$, satisfying

$$
r_{h}=2 M+\frac{1}{3} \Lambda r_{h}^{3} \leq 2 M
$$

in terms of which

$$
f=1-\frac{\left(1-\frac{1}{3} \Lambda r_{h}^{2}\right) r_{h}}{r}-\frac{\Lambda r^{2}}{3}
$$

These black holes have a non-static region I defined by $0<r<r_{h}$ and a static region II defined by $r_{h}<r<\infty$.

The SdS black holes are those for which $0<\Lambda<(3 M)^{-2}\left(f\right.$ has single real root when $\Lambda>(3 M)^{-2}$, and this root is negative). For SdS black holes $f$ has one negative $\left(r_{o}\right)$ and two positive roots $r_{h}<r_{c}, r_{o}+r_{h}+r_{c}=0$, with $r_{c} \rightarrow r_{h}+$ as $9 M^{2} \Lambda \rightarrow 1^{-}$, and $[22]$

$$
2 M<r_{h}<3 M<r_{c}
$$

There is a non-static region I defined by $0<r<r_{h}$ adjacent to a static region II $\left(r_{h}<r<r_{c}\right)$, and a further non-static region III defined by $r>r_{c}$.

This paper focuses on the stability of the static region II of Schwarzschild and S(A)dS black holes.

2. The bifurcation sphere at $r=r_{h}$

Let $g$ be the function

$$
g(r)=\frac{1}{f(r)}-\frac{1}{f^{\prime}\left(r_{h}\right)\left(r-r_{h}\right)} .
$$

$g$ is smooth in I $\cup$ II (i.e., $0<r<r_{c}$ for SdS, $0<r$ for Schwarzschild and SAdS), since $f$ is smooth with a simple zero at $r=r_{h}$ and no zeros in I and II.

Consider the following solution of (48)

$$
r^{*}=G(r)+\frac{1}{f_{h}^{\prime}} \ln \left|\frac{r}{r_{h}}-1\right|, \quad \frac{d G}{d r}=g, \quad f_{h}^{\prime}=f^{\prime}\left(r_{h}\right)
$$

The most general solution is obtained by adding (possibly different) constants to the left and right of $r=r_{h}$, however, for $r^{*}$ as in (55), the function

$$
s(r)=\left(r / r_{h}-1\right) e^{f_{h}^{\prime} G}= \begin{cases}e^{f_{h}^{\prime} r^{*}} & , \text { in II } \\ -e^{f_{h}^{\prime} r^{*}} & , \text { in I }\end{cases}
$$

is smooth in $\mathrm{I} \cup \mathrm{II}$, and monotonically growing, so it has an inverse $r=\mathcal{K}(s)$. Introduce $u=t-r^{*}$ in addition to $v=t+r^{*}$ above, then

$$
d s^{2}=-f(r) d u d v+r^{2}\left(d \theta^{2}+\sin ^{2} \theta d \phi^{2}\right)
$$


Now let

$$
(U, V)= \begin{cases}\left(-e^{-f_{h}^{\prime} u / 2}, e^{f_{h}^{\prime} v / 2}\right) & , \text { in II } \\ \left(e^{-f_{h}^{\prime} u / 2}, e^{f_{h}^{\prime} v / 2}\right) & , \text { in I }\end{cases}
$$

Note that $U V=-s(r)$ and $d U d V=\frac{1}{4} f_{h}^{\prime 2} s(r) d u d v$, therefore (47) is equivalent to

$$
d s^{2}=\frac{4 f(r)}{f_{h}^{\prime 2} U V} d U d V+r^{2}\left(d \theta^{2}+\sin ^{2} \theta d \phi^{2}\right), \quad r=k(-U V), \quad k(-U V)>0
$$

and $V>0$. We may now take two extra copies of I and II (call these $\mathrm{I}^{\prime}$ and $\mathrm{II}^{\prime}$ ) and define

$$
(U, V)= \begin{cases}\left(e^{-f_{h}^{\prime} u / 2},-e^{f_{h}^{\prime} v / 2}\right) & , \text { in } \mathrm{II}^{\prime} \\ \left(-e^{-f_{h}^{\prime} u / 2},-e^{f_{h}^{\prime} v / 2}\right) & , \text { in } \mathrm{I}^{\prime}\end{cases}
$$

The black hole metric (47) in $\mathrm{I}^{\prime} \cup \mathrm{II}^{\prime}$ is again given by equation (59), except that now $V<0$. It can be checked that (59) is smooth on $S^{2}$ times the open region of the $(U, V)$ plane defined by $\mathcal{K}(-U V)>0\left(0<\mathcal{K}(-U V)<r_{c}\right.$ for $\left.\mathrm{SdS}\right)$. This region contains two copies of I and two copies of II. Since $r$ is a function of the product $U V$, the metric (59) has the discrete $Z_{2}$ symmetry $(U, V) \rightarrow(-U,-V)$ under which $\mathrm{I} \leftrightarrow \mathrm{I}^{\prime}$ and $\mathrm{II} \leftrightarrow \mathrm{II}^{\prime}$. The $Z_{2}$ invariant set $U=V=0$ is a sphere of radius $r_{h}$, called bifurcation sphere.

Integrating (48) in region II we find that, for $\Lambda=0$, after choosing an integration constant,

$$
r^{*}=r+2 M \ln \left(\frac{r}{2 M}-1\right) ;
$$

for $\Lambda<0$ the integration constant can be chosen such that

$$
r^{*}=-\int_{r}^{\infty} \frac{d r^{\prime}}{f\left(r^{\prime}\right)} \simeq\left\{\begin{array}{ll}
\frac{r_{h}}{1-\Lambda r_{h}^{2}} \ln \left(\frac{r}{r_{h}}-1\right) & , r \rightarrow r_{h}^{+} \\
\frac{3}{\Lambda r} & , r \rightarrow \infty
\end{array},\right.
$$

and for $0<9 M^{2} \Lambda<1$ and $r_{h}<r<r_{c}$,

$$
r^{*} \simeq \begin{cases}\frac{r_{h}\left(r_{h} r_{c}+r_{h}{ }^{2}+r_{c}^{2}\right)}{\left(r_{c}-r_{h}\right)\left(2 r_{h}+r_{c}\right)} \ln \left(\frac{r}{r_{h}}-1\right) & , r \rightarrow r_{h}^{+} \\ \frac{r_{c}\left(r_{h} r_{c}+r_{h}{ }^{2}+r_{c}^{2}\right)}{\left(r_{h}-r_{c}\right)\left(2 r_{c}+r_{h}\right)} \ln \left(\frac{r}{r_{c}}-1\right) & , r \rightarrow r_{c}^{-}\end{cases}
$$

It follows that, in region II of a Schwarzschild or SdS black hole, $-\infty<r^{*}<\infty$ (which corresponds to the entire quadrants $U<0, V>0$ and $U>0, V<0)$. For SAdS, on the other hand, $-\infty<r^{*}<0$, therefore $U>-1 / V$ in II, and $U<-1 / V$ in $\mathrm{II}^{\prime}$.

The above construction gives the Penrose-Carter diagrams in Figure 1. In the AdS case (right side of the figure), there is a conformal time like boundary corresponding to $U>-1 / V$ in II, and $U<-1 / V$ in $\mathrm{II}^{\prime}$. The conformal boundary of region II is replaced in the Schwarzschild case by null infinity, and in the SdS case by the cosmological horizon (left side of the figure). For the SdS black hole it is possible to follow a procedure similar to the one outlined above that gives a further extension with a bifurcation sphere at $r=r_{c}$, two copies of region III, and an extra copy of II. The maximal analytic extension of SdS contains infinitely many copies of I, II, and III [15].

\section{Hyperbolic equations and global hyperbolicity of the static region}

If we use the coordinates $\left(t, r^{*}, \theta, \phi\right)$, the scalar wave equation (22) acquires the simple form

$$
\nabla^{\alpha} \nabla_{\alpha} \Phi=\frac{1}{r f}\left[-\partial_{t}^{2}+\partial_{r^{*}}^{2}+f\left(\frac{\widehat{D}^{A} \widehat{D}_{A}}{r^{2}}-\frac{2 M}{r^{3}}+\frac{2 \Lambda}{3}\right)\right](r \Phi),
$$

and (4DRWE) reduces to

$$
0=-r f\left[\nabla^{\alpha} \nabla_{\alpha} \Phi+\left(\frac{8 M}{r^{3}}-\frac{2 \Lambda}{3}\right) \Phi\right]=\left[\partial_{t}^{2}-\partial_{r^{*}}^{2}-f\left(\frac{\widehat{D}^{A} \widehat{D}_{A}}{r^{2}}+\frac{6 M}{r^{3}}\right)\right](r \Phi)
$$



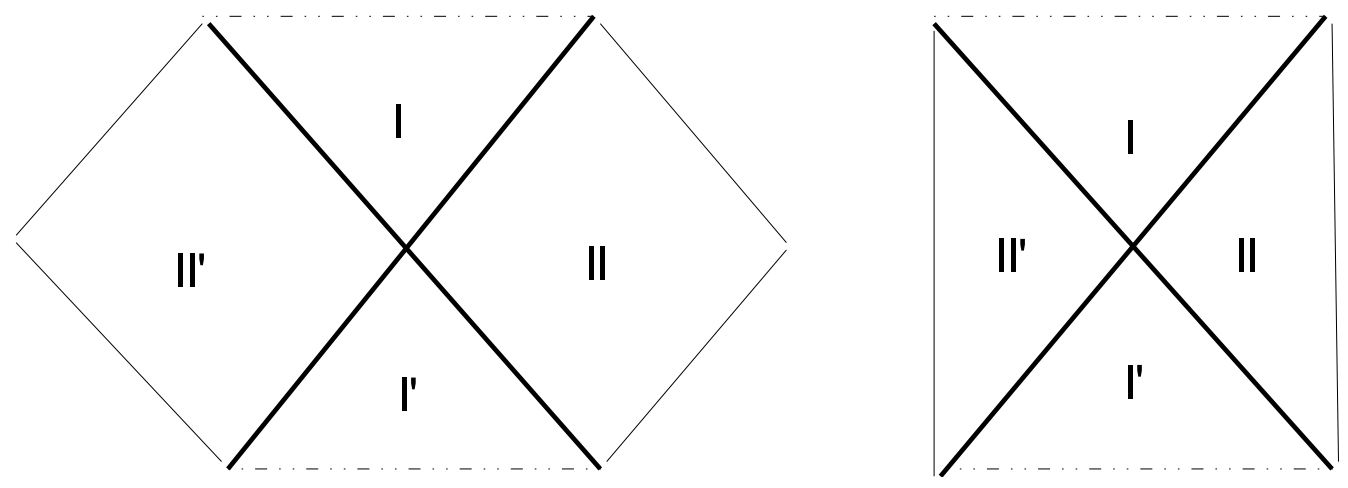

FIG. 1. Left: Penrose-Carter diagram for a Schwarzschild black hole (SdS black hole). The dotted lines are the $r=0$ singularities, the thick lines are past and future event horizons -the $U$ and $V$ axis in (59)-, they intersect at the bifurcation sphere. The thin lines correspond to null infinity (the cosmological horizon).

Right: Penrose-Carter diagram for a SAdS black hole. The dotted lines are the singularities at $r=0$, the thick lines are the past and future event horizons -the $U$ and $V$ axis intersecting at the bifurcation sphere-. The thin time-like lines correspond to null infinity.

that is,

$$
\frac{\partial^{2}}{\partial t^{2}}(r \Phi)+\mathcal{A}^{\Lambda}(r \Phi)=0
$$

where

$$
\mathcal{A}^{\Lambda}=-\frac{\partial^{2}}{\partial r^{* 2}}+\left(1-\frac{2 M}{r}-\frac{\Lambda}{3} r^{2}\right)\left(-\frac{6 M}{r^{3}}-\frac{\widehat{D}^{A} \widehat{D}_{A}}{r^{2}}\right)=:-\frac{\partial^{2}}{\partial r^{* 2}}+V_{1}-V_{2} \widehat{D}^{A} \widehat{D}_{A},
$$

and

$$
V_{1}=\left(1-\frac{2 M}{r}-\frac{\Lambda}{3} r^{2}\right)\left(-\frac{6 M}{r^{3}}\right) \quad \text { and } \quad V_{2}=\left(1-\frac{2 M}{r}-\frac{\Lambda}{3} r^{2}\right)\left(\frac{1}{r^{2}}\right) .
$$

If we expand $\Phi$ in spherical harmonics,

$$
\Phi=\sum_{(\ell \geq 0, m)} \frac{\phi_{(\ell, m)}}{r} S_{(\ell, m)}: \mathcal{M} \rightarrow \mathbb{R}
$$

and use this in equations (66)-(68), we find that (4DRWE) is indeed equivalent to the set of equations (RWE), extended to include $\ell=0,1$.

Note that, although the above equations are formally similar for different values of $\Lambda,-\infty<r^{*}<\infty$ as $r$ spans region II of the Schwarzschild and SdS black holes, but is restricted to $r^{*}<0$ in region II of the SAdS black hole. The reason why equation (4DRWE) for SAdS is equivalent to a system of wave equations with a potential in $a$ half of a two dimensional Minkowski space, is that region II of SAdS is not globally hyperbolic, but has a conformal timelike boundary at $r^{*}=0$ (equation (62)). The problem of defining the dynamics imposed by hyperbolic equations (such as (4DRWE), (RWE) and (ZE)) in a non globally hyperbolic static spacetime (either SAdS or its two dimensional orbit space SAdS $/ S O(3)$ ), that is, giving unique solutions from initial data at a surface $\Sigma_{o}$ transverse to the timelike Killing vector field $k^{a}$, has been addressed in the series of articles [31], [19] and [20]. As expected, the dynamics is unique only within the domain of dependence of the support of the initial datum; outside it, it depends on the self adjoint extension that we choose for the operator $\mathcal{A}$ which is roughly our choice of boundary conditions at $r^{*}=0$. 
For the Zerilli equation (ZE) of an SAdS black hole, we have found that there is a choice of boundary conditions at $r^{*}=0$ under which even perturbations that preserve the AdS asymptote, grow exponentially with time. For this choice, SAdS is unstable. There is an infinite set of possible boundary conditions for equation (ZE) in SAdS/SO(3), we are currently analyzing the dynamics and stability of SAdS black holes under different choices [33].

\section{THE LINEARIZED EINSTEIN EQUATION: ODD SECTOR}

There are many sources for the well know solution of the LEE around a Schwarzschild background. Two historically relevant references are [23], where the gauge invariance of RW and Zerilli potentials was established, and [12], where a "covariant" formulation using arbitrary coordinates for the orbit space in order to study, e.g., waves crossing the horizon was developed (this should more properly be called "2D covariant approach"). More recently, the 2D covariant approach was generalized to GR black holes with constant curvature horizons in arbitrary dimensions and including a cosmological constant in a series of papers by Ishibashi and Kodama (see, e.g., [13] and [14]). We find the 2D covariant approach in reference [27] particularly well adjusted to our purposes as, although restricted to the dynamical $\ell \geq 2$ perturbations, avoids unnecessary expansions in harmonic modes. Section V in [27] is devoted to the Schwarzschild black hole, we generalize the exposition there to $\Lambda \neq 0$ below.

\section{A. Gauge transformations and gauge invariants}

The effect of the diffeomorphism generated by the vector field $\xi^{\alpha}$ on the first order variation of a tensor $T$ is $\dot{T} \rightarrow \dot{T}+£_{\xi} T$. In particular, the effect on $h_{\alpha \beta}=\dot{g}_{\alpha \beta}$,

$$
h_{\alpha \beta} \rightarrow £_{\xi} g_{\alpha \beta}=h_{\alpha \beta}+\nabla_{\alpha} \xi_{\beta}+\nabla_{\beta} \xi_{\alpha}=h_{\alpha \beta}^{\prime},
$$

defines an equivalence relation where $h_{\alpha \beta} \sim h_{\alpha \beta}^{\prime}$ iff there exists a covector field $\xi_{\alpha}$ such that (70) holds. To measure the strength of the perturbation we will analyze gauge invariant fields, i.e., fields that depend only on the class $\left[h_{\alpha \beta}\right]$ of a metric perturbation under the equivalence relation defined above. In this section we show how to parametrize the set of classes $\left[h_{\alpha \beta}\right]$ by means of gauge invariant fields and how to choose a class representative (gauge fixing).

Given a generic vector field (26)-(30) we find that

$$
\nabla_{\alpha} \xi_{\beta}^{(-)}+\nabla_{\beta} \xi_{\alpha}^{(-)}=\left(\begin{array}{cc}
0 & \widehat{\epsilon}_{B}^{C} \widehat{D}_{C}\left(r^{2} \widetilde{D}_{a} Y\right) \\
\widehat{\epsilon}_{A}{ }^{C} \widehat{D}_{C}\left(r^{2} \widetilde{D}_{b} Y\right) & 2 r^{2} \widehat{\epsilon}_{(B}{ }^{C} \widehat{D}_{A)} \widehat{D}_{C} Y
\end{array}\right)
$$

and

$$
\nabla_{\alpha} \xi_{\beta}^{(+)}+\nabla_{\beta} \xi_{\alpha}^{(+)}=\left(\begin{array}{cc}
\widetilde{D}_{a} \xi_{b}+\widetilde{D}_{b} \xi_{a} & \widehat{D}_{B}\left(r^{2} \widetilde{D}_{a} X+\xi_{a}\right) \\
\widehat{D}_{A}\left(r^{2} \widetilde{D}_{b} X+\xi_{b}\right) & 2 r^{2} \widehat{D}_{A} \widehat{D}_{B} X+2 r\left(\widetilde{D}_{a} r\right) \xi^{a} \widehat{g}_{A B}
\end{array}\right)
$$

where it should be kept in mind that $X$ and $Y$ belong to $L^{2}\left(S^{2}\right)_{>0}$. These equations imply that the odd (even) piece of $\xi_{\alpha}$ affects the odd (even) piece of $h_{\alpha \beta}$ in (70). Let us analyze the effect of gauge transformations in the odd sector. Inserting $Y=Y_{(\ell=1)}+Y^{>1}$,

$$
Y_{(\ell=1)}=\sum_{m=1}^{3} Y_{(m)}(x) S_{(\ell=1, m)}(\theta, \phi), \quad Y^{>1}=\sum_{\ell \geq 2, m}^{3} Y_{(\ell, m)}(x) S_{(\ell=1, m)}(\theta, \phi),
$$

in (71) ( $x$ are coordinates of the orbit manifolds, $S_{(\ell, m)}$ orthonormal real spherical harmonics), defining $h_{\alpha \beta}^{(-,>1)}=$ $h_{\alpha \beta}^{(-)}-h_{\alpha \beta}^{(\ell=1,-)}$ and splitting similarly all the other fields, we find from (43), (70) and (40) that

$$
h_{\alpha \beta}^{\prime(\ell=1,-)}=\left(\begin{array}{cc}
0 & \sum_{m=1}^{3} \sqrt{\frac{3}{4 \pi}}\left(h_{a}^{(\ell=1, m)}+r^{2} \widetilde{D}_{a} Y_{(m)}\right) J_{(m) B} \\
\sum_{m=1}^{3} \sqrt{\frac{3}{4 \pi}}\left(h_{b}^{(\ell=1, m)}+r^{2} \widetilde{D}_{b} Y_{(m)}\right) J_{(m) A} & 0
\end{array}\right)
$$


and also that

$$
h_{\alpha \beta}^{\prime(-,>1)}=\left(\begin{array}{cc}
0 & \widehat{\epsilon}_{B}{ }^{C} \widehat{D}_{C}\left(h_{a}^{>1}+r^{2} \widetilde{D}_{a} Y^{>1}\right) \\
\widehat{\epsilon}_{A}{ }^{C} \widehat{D}_{C}\left(h_{b}^{>1}+r^{2} \widetilde{D}_{b} Y^{>1}\right) & 2 r^{2} \widehat{\epsilon}_{(A}{ }^{C} \widehat{D}_{B)} \widehat{D}_{C}\left(k^{>1}+Y^{>1}\right)
\end{array}\right)
$$

This last equation implies that: i) $H_{a}=h_{a}^{>1}-r^{2} \widetilde{D}_{a} k^{>1}$ is a gauge invariant field and ii) there is a gauge (commonly known as the Regge-Wheeler gauge) where the $\ell>1$ piece of the odd metric perturbation assumes the form

$$
h_{\alpha \beta}^{(-,>1)}=\left(\begin{array}{cc}
0 & \widehat{\epsilon}_{B}^{C} \widehat{D}_{C} H_{a} \\
\widehat{\epsilon}_{A}{ }^{C} \widehat{D}_{C} H_{b} & 0
\end{array}\right), \quad H_{a} \in L^{2}\left(S^{2}\right)_{>1}
$$

\section{B. Solution of the linearized Einstein equation}

A calculation of the LEE (1) using (75) gives, in agreement with equations (31) in [5],

$$
\begin{aligned}
\dot{R}_{a b}-\Lambda h_{a b} & =0 \\
\dot{R}_{a B}-\Lambda h_{a B} & =-\frac{1}{2 r^{2}} \widehat{\epsilon}_{B}{ }^{C} \widehat{D}_{C}\left[\tilde{\epsilon}_{a}^{c} \widetilde{D}_{c}\left(r^{2} \mathcal{F}\right)-H_{a} \widetilde{D}^{c} \widetilde{D}_{c}\left(r^{2}\right)+\widehat{D}^{C} \widehat{D}_{C} H_{a}+2 \Lambda r^{2} H_{a}\right] \\
\dot{R}_{A B}-\Lambda h_{A B} & =\widehat{\epsilon}_{(A}{ }^{C} \widehat{D}_{B)} \widehat{D}_{C}\left(\widetilde{D}^{a} H_{a}\right)
\end{aligned}
$$

where

$$
\mathcal{F}=r^{2} \tilde{\epsilon}^{a b} \widetilde{D}_{a}\left(\frac{H_{b}}{r^{2}}\right)
$$

In deriving equations (76)-(79) we have not made any assumptions on $H_{a}$ in (75), so these calculations also hold for the $\ell=1$ mode given in (43) and (40) if we replace $H_{a}$ with $\sum_{m} h_{a}^{(\ell=1, m)}(x) S_{(1, m)}$. In this case, however,

$$
\left(-\widetilde{D}^{c} \widetilde{D}_{c}\left(r^{2}\right)+\widehat{D}^{C} \widehat{D}_{C}+2 \Lambda r^{2}\right) h_{a}^{(\ell=1, m)} S_{(1, m)}=0
$$

and also $\widehat{\epsilon}_{(A}{ }^{C} \widehat{D}_{B)} \widehat{D}_{C}\left(S_{(1, m)}\right)=0$, so the LEE for the odd $\ell=1$ modes can entirely be written in terms of the field

$$
\mathcal{F}_{1}=r^{2} \tilde{\epsilon}^{a b} \widetilde{D}_{a}\left(\sum_{m} \frac{h_{b}^{(\ell=1, m)}}{r^{2}}\right) S_{(1, m)}
$$

which is invariant under the gauge transformation (73). Thus, for the odd $\ell=1$ mode we find

$$
\begin{aligned}
\dot{R}_{a b}-\Lambda h_{a b} & =0 \\
\dot{R}_{a B}-\Lambda h_{a B} & =-\frac{1}{2 r^{2}} \widehat{\epsilon}_{B}{ }^{C} \widehat{D}_{C}\left[\tilde{\epsilon}_{a}^{c} \widetilde{D}_{c}\left(r^{2} \mathcal{F}_{1}\right)\right] \\
\dot{R}_{A B}-\Lambda h_{A B} & =0 .
\end{aligned}
$$

Using the facts that the kernel of the operator $\widehat{\epsilon}_{A}{ }^{C} \widehat{D}_{C}$ is the $\ell=0$ mode and the kernel of $\widehat{\epsilon}_{(A}{ }^{C} \widehat{D}_{B)} \widehat{D}_{C}$ are the $\ell=0,1$ modes, we arrive at

Lemma 3. The LEE (1) in the odd sector is equivalent to the system

$$
\begin{aligned}
& \widetilde{D}^{a} H_{a}=0 \\
& \tilde{\epsilon}_{b}^{c} \widetilde{D}_{c}\left(r^{2} \mathcal{F}\right)-H_{b} \widetilde{D}^{c} \widetilde{D}_{c}\left(r^{2}\right)+\widehat{D}^{C} \widehat{D}_{C} H_{b}+2 \Lambda r^{2} H_{b}=0 \\
& \widetilde{D}_{c}\left(r^{2} \mathcal{F}_{1}\right)=0 .
\end{aligned}
$$


Let us treat first the equations for $\ell=1$ modes. The general solution of equation (86) is [27]

$$
h_{a}^{(\ell=1, m)}=\frac{2 j^{(m)}}{r N(r)} \tilde{\epsilon}_{a}{ }^{b} \widetilde{D}_{b} r+r^{2} \widetilde{D}_{a}\left(\frac{Z^{(m)}}{r^{2}}\right), N(r)=g^{a b}\left(\widetilde{D}_{a} r\right)\left(\widetilde{D}_{b} r\right),
$$

$j^{(m)}$ a gauge invariant constant and $Z^{(m)}$ an arbitrary function that we recognize from (73) as "pure gauge". For the Schwarzschild-(A)dS space-time in Schwarzschild coordinates, $g_{a b} d x^{a} d x^{b}=-f(r) d t^{2}+d r^{2} / f(r), f(r)=1-2 M / r-$ $\Lambda r^{2} / 3=N(r)$, and equation (87) reads

$$
h_{a}^{(\ell=1, m)} d x^{a}=-\frac{2 j^{(m)}}{r} d t+r^{2} \widetilde{D}_{a}\left(\frac{Z^{(m)}}{r^{2}}\right) d x^{a} .
$$

If we take, e.g., $j^{(1)}=j^{(2)}=0, j^{(3)}=a M$, choose the gauge $Z^{(1)}=Z^{(2)}=0, Z^{(3)}=-\Lambda(a / 3) r^{2} t$, and insert the above equation in (43), we obtain

$$
h_{\alpha \beta}^{(\ell=1,-)} d x^{\alpha} d x^{\beta}=-2\left(\frac{2 a M}{r}+\frac{\Lambda a}{3} r^{2}\right) \sin ^{2}(\theta) d t d \phi,
$$

which we recognize as the first order Taylor expansion around $a=0$ of the Kerr-(A)dS metric with angular momentum $J=a M$ in Boyer-Lindquist coordinates. This proves our previous assertion that $\ell=1$ odd modes correspond to displacements within the stationary Kerr family of black holes.

Consider now the $\ell>1$ odd LEE, equations (84) and (85). For the Schwarzschild-(A)dS background equation (85) reads

$$
\mathcal{E}_{b}=\tilde{\epsilon}_{b}{ }^{c} \widetilde{D}_{c}\left(r^{2} \mathcal{F}\right)+\left(2+\widehat{D}^{A} \widehat{D}_{A}\right) H_{b}=0,
$$

and, as noticed in [5], implies (84) (proof: $0=\widetilde{D}^{b} \mathcal{E}_{b}=\left(2+\widehat{D}^{A} \widehat{D}_{A}\right) \widetilde{D}^{b} H_{b}$ implies $\widetilde{D}^{b} H_{b}=0$ since $\left.H_{b} \in L^{2}\left(S^{2}\right)_{>1}\right)$. On the other hand, equation (90) implies (but is not equivalent to!) $\tilde{\epsilon}^{a b} \widetilde{D}_{a}\left(r^{-2} \mathcal{E}_{b}\right)=0$, a condition that, from the definition of $\mathcal{F}$, equations (22) and (79) and the background equation

$$
\widetilde{D}_{a} \widetilde{D}_{b} r=\left(\frac{M}{r^{2}}-\frac{\Lambda}{3} r\right) \tilde{g}_{a b}
$$

is seen to be equivalent to

$$
\nabla^{\alpha} \nabla_{\alpha} \mathcal{F}+\left(\frac{8 M}{r^{3}}-\frac{2 \Lambda}{3}\right) \mathcal{F}=0
$$

Interestingly enough, (86) and (80) imply that $\mathcal{F}_{1}=\sum_{m=1}^{3} C_{(m)} S_{(1, m)}(\theta, \phi) / r^{2}$ which, using (22) is easily seen to also satisfy equation (92). We conclude that the LEE implies that

$$
\nabla^{\alpha} \nabla_{\alpha} F+\left(\frac{8 M}{r^{3}}-\frac{2 \Lambda}{3}\right) F=0, \quad\left(F=\mathcal{F}_{1}+\mathcal{F}\right) .
$$

Back to (84)-(85), we note that the solution of (84) is

$$
H_{a}=\tilde{\epsilon}_{a b} \widetilde{D}^{b}\left(r^{2} \Phi\right)
$$

for some scalar field $\Phi \in L^{2}\left(S^{2}\right)_{>1}$. This implies that

$$
\mathcal{F}=r^{2} \widetilde{D}^{c}\left(r^{-2} \widetilde{D}_{c}\left(r^{2} \Phi\right)\right) .
$$

Inserting (94) in (90) gives

$$
\tilde{\epsilon}_{b}{ }^{c} \widetilde{D}_{c}\left[r^{2} \mathcal{F}+\left(2+\widehat{D}^{A} \widehat{D}_{A}\right) r^{2} \Phi\right]=0 .
$$

The general solution to this equation is

$$
\frac{\mathcal{F}}{r^{2}}+\frac{2+\widehat{D}^{A} \widehat{D}_{A}}{r^{2}} \Phi=\widetilde{D}^{c}\left(r^{-2} \widetilde{D}_{c}\left(r^{2} \Phi\right)\right)+\frac{2+\widehat{D}^{A} \widehat{D}_{A}}{r^{2}} \Phi=\frac{z(\theta, \phi)}{r^{4}},
$$


and we may (and will) choose $z(\theta, \phi)=0$, which gives

$$
\mathcal{F}=-\left[2+\widehat{D}^{A} \widehat{D}_{A}\right] \Phi=-\left[2+\mathbf{J}^{2}\right] \Phi,
$$

together with

$$
\begin{aligned}
0 & =\widetilde{D}^{c}\left(r^{-2} \widetilde{D}_{c}\left(r^{2} \Phi\right)\right)+\frac{2+\widehat{D}^{A} \widehat{D}_{A}}{r^{2}} \Phi \\
& =\widetilde{D}^{c} \widetilde{D}_{c} \Phi+\frac{\widehat{D}^{A} \widehat{D}_{A}}{r^{2}} \Phi+\frac{2}{r} \widetilde{D}^{c} r \widetilde{D}_{c} \Phi+\frac{2}{r^{2}}\left(1-\widetilde{D}^{c} r \widetilde{D}_{c} r+r \widetilde{D}^{c} \widetilde{D}_{c} r\right) \Phi,
\end{aligned}
$$

which, using again (22) and (91), is seen to be equivalent to the 4DRW equation

$$
\nabla^{\alpha} \nabla_{\alpha} \Phi+\left(\frac{8 M}{r^{3}}-\frac{2 \Lambda}{3}\right) \Phi=0, \quad \Phi \in L^{2}\left(S^{2}\right)_{>1} .
$$

Equation (100) is equivalent to the standard form (RWE) after decomposing $\Phi$ in modes as in (19). We can gather our results concerning the odd sector of the LEE in the following:

Lemma 4. Consider the odd sector of the LEE around a Schwarzschild-(A)dS background:

(i) The solution of the $\ell>1$ piece of the metric perturbation in the Regge-Wheeler gauge is given by equation (75), where the gauge invariant field $H_{a}$ is as in equation (94) and $\Phi$ satisfies the $4 D R W$ equation (100). Equations (75) and (94) are equivalent to (18).

(ii) The solution of the $\ell=1$ piece is given in equations (43) and (88). In this last equation $j^{(m)}$ are three gauge invariant constants and $Z^{(m)}$ three arbitrary gauge functions.

(iii) There is a bijection between the space $\mathcal{L}_{-}$of smooth odd solutions of the LEE mod gauge transformations and the set

$$
L_{-}=\left\{j^{(m)}, m=1,2,3\right\} \cup\{\Phi \mid \Phi \text { is a smooth solution of equation (100) }\} .
$$

(iv) The LEE implies that the gauge invariant field $F=\mathcal{F}_{1}+\mathcal{F}$ (defined in equations (79) and (80)) satisfies the $4 D R W$ equation (93).

The equivalence of (75) and (94) to (18) is checked by a straightforward calculation. (iii) follows immediately from (i) and (ii). Note also that the relation (98) explains why the fields $\mathcal{F}$ and $\Phi$, which belong to $L^{2}\left(S^{2}\right)_{>1}$, satisfy the same wave equation: the operators $\nabla^{\alpha} \nabla_{\alpha}+\left(\frac{8 M}{r^{3}}-\frac{2 \Lambda}{3}\right)$ and $\left[2+\widehat{D}^{A} \widehat{D}_{A}\right]$ commute, and $\left[2+\widehat{D}^{A} \widehat{D}_{A}\right]$ is invertible in $L^{2}\left(S^{2}\right)_{>1}$ (on scalar fields, $\widehat{D}^{A} \widehat{D}_{A}=\mathbf{J}^{2}$ and on arbitrary tensor fields $\left[\mathbf{J}^{2}, \nabla_{\alpha}\right]=0$, since $\left[£_{K}, \nabla_{\alpha}\right]=0$ for any Killing vector field $\left.K^{\alpha}\right)$.

\section{Measurable effects of the perturbation on the geometry}

For the purposes of a non-modal analysis, Lemma 4.(iii) offers a more appropriate parametrization of the dynamical sector of the odd perturbations than the infinite set of $\phi_{(\ell, m)}^{-}$'s. However, unlike the $\ell=1$ parameters $j_{(m)}$, no clear geometrical meaning can be attached to $\Phi$ in (100), beyond that of being a potential for solutions of the LEE, equation (18). Consider now the curvature scalars in (7). In this section we show that the first order variation $G_{-}=\dot{Q}_{-}$of $Q_{-}$associated to a perturbation $\left[h_{\alpha \beta}^{(-)}\right] \in \mathcal{L}_{-}$contains all the gauge invariant information about $h_{\alpha \beta}^{(-)}$. In particular, $\Phi$ and the $j^{(m)}$ can be recovered from $G_{-}=\dot{Q}_{-}$which, unlike $\Phi$, has a distinct geometrical meaning. Note that, since $Q_{-}=0$ for the $\mathrm{S}(\mathrm{A}) \mathrm{dS}$ geometries, $\dot{Q}_{-}$is gauge invariant.

$\dot{Q}_{-}$can be obtained from the set of equations (3) and (29) in [5]. The calculations are tedious and not particularly illuminating. The result is

$$
\dot{Q}_{-}=\frac{3 M}{r^{5}} \widehat{D}^{A} \widehat{D}_{A} F=\frac{3 M}{r^{5}} \mathbf{J}^{2} F, \quad\left(F=\mathcal{F}_{1}+\mathcal{F}\right)
$$


As an example, for the choice $j^{(1)}=j^{(2)}=0, j^{(3)}=a M$ in (89), the $\ell=1$ piece of $\dot{Q}$ in (101) is

$$
\dot{Q}_{-}^{(\ell=1)}=\frac{3 M}{r^{5}} \mathbf{J}^{2} \mathcal{F}_{1}=-\frac{6 M^{2} a}{r^{7}} \cos \theta,
$$

which agrees with the first order Taylor expansion of $Q_{-}$for the Kerr-(A)de-Sitter black hole in Boyer-Lindquist coordinates around $a=0$, as anticipated. For arbitrary $j^{(m)}$ 's, equation (102) generalizes to

$$
\dot{Q}_{-}^{(\ell=1)}=-\frac{6 M}{r^{7}} \sqrt{\frac{4 \pi}{3}} \sum_{m=1}^{3} j^{(m)} S_{(1, m)} .
$$

Combining equations (98), (101) and (103) gives

$$
\dot{Q}_{-}=-\frac{6 M}{r^{7}} \sqrt{\frac{4 \pi}{3}} \sum_{m=1}^{3} j^{(m)} S_{(1, m)}-\frac{3 M}{r^{5}} \mathbf{J}^{2}\left(\mathbf{J}^{2}+2\right) \Phi,
$$

which generalizes to $\Lambda \neq 0$ equation (22) in [9].

Theorem 1. Let $\left[h_{\alpha \beta}^{(-)}\right] \in \mathcal{L}_{-}$and $\dot{Q}_{-}\left(\left[h_{\alpha \beta}^{(-)}\right]\right)$be the first order variation of $Q_{-}$for the perturbation $\left[h_{\alpha \beta}^{(-)}\right]$:

(i) The field $r^{5} \dot{Q}_{-}$is in $L^{2}\left(S^{2}\right)_{>0}$ and satisfies the $4 D R W$ equation

$$
\left[\nabla^{\alpha} \nabla_{\alpha}+\left(\frac{8 M}{r^{3}}-\frac{2 \Lambda}{3}\right)\right]\left(r^{5} \dot{Q}_{-}\right)=0
$$

(ii) The map $\left[h_{\alpha \beta}^{(-)}\right] \rightarrow \dot{Q}_{-}\left(\left[h_{\alpha \beta}^{(-)}\right]\right)$is invertible: it is possible to construct a representative of $\left[h_{\alpha \beta}^{(-)}\right]$from $\dot{Q}_{-}\left(\left[h_{\alpha \beta}^{(-)}\right]\right)$.

Proof.

(i) Equation (104) proves that $\dot{Q}_{-} \in L^{2}\left(S^{2}\right)_{>0}$. This is a consequence of the facts that there are no odd $\ell=0$ modes, only odd modes contribute to $\dot{Q}_{-}$, and $\mathbf{J}^{2} \dot{Q}_{-}\left(\left[h_{\alpha \beta}^{(-)}\right]\right)=\dot{Q}_{-}\left(\mathbf{J}^{2}\left[h_{\alpha \beta}^{(-)}\right]\right)$. Alternatively, by Birkhoff's theorem in a cosmological background [28], the only possible spherically symmetric perturbation of a Schwarzschild-(A)dS black hole amounts to a change of the black hole mass, and this does not affect the unperturbed value $Q_{-}=0$. Since $\mathbf{J}^{2}$ commutes with the wave operator in (93), it follows from (101) and Lemma 4.(iv) that $r^{5} \dot{Q}_{-}$satisfies (105).

(ii) According to equation (104), from the $\ell=1$ coefficients in the spherical harmonic expansion of $\dot{Q}_{-}$we obtain the constants $j^{(m)}$. This allows us to construct the $\ell=1$ piece of the $Z^{(m)}=0$ representative of $\left[h_{\alpha \beta}^{(-)}\right]$in $(87)$ and use it in (43). The orthogonal projection of $\frac{r^{5}}{3 M} \dot{Q}_{-}$onto $L^{2}\left(S^{2}\right)_{>1}$ is related to $\Phi$ through the operator $\mathbf{J}^{2}\left(\mathbf{J}^{2}+2\right)$ (equation (104)). This operator is invertible in $L^{2}\left(S^{2}\right)_{>1}$, so we can recover $\Phi$ from $\dot{Q}_{-}\left(\left[h_{\alpha \beta}^{(-)}\right]\right)$and use it in (94) or (18) to construct the Regge-Wheeler representative (75) of the $\ell>1$ piece of $\left[h_{\alpha \beta}^{(-)}\right]$.

Equation (104) defines a bijection between the set $L_{-} \sim \mathcal{L}_{-}$defined in Lemma 4.(iii) and the set

$$
\hat{\mathcal{L}}_{-}=\left\{\dot{Q}_{-}\left(\left[h_{\alpha \beta}^{(-)}\right]\right) \mid h_{\alpha \beta}^{(-)} \text {is a solution of the LEE }\right\}
$$

This bijection implies that $\dot{Q}_{-}$contains all the relevant (i.e., gauge invariant) information on the metric perturbation that gave rise to it. The invertible relations

$$
\Phi \stackrel{-\left(2+\mathbf{J}^{2}\right)}{\longrightarrow} \mathcal{F} \stackrel{\mathbf{J}^{2}}{\longrightarrow} r^{5} \dot{Q}_{-}^{>1}
$$

and

$$
F \stackrel{\mathrm{J}^{2}}{\rightarrow} r^{5} \dot{Q}_{-},
$$

explain why all these fields obey the same way equation. Note that the addition of an $\ell=1$ piece $\Phi^{(\ell=1)}$ to the potential $\Phi$ satisfying a 4 DRW equation would be irrelevant, as its contribution to $H_{a}$ would vanish: $\tilde{\epsilon}_{a b} \widetilde{D}^{b}\left(r^{2} \Phi^{(\ell=1)}\right)=0$. 


\section{Nonmodal linear stability of the $\Lambda \geq 0$ black holes}

Having found that the scalar gauge invariant field $G_{-}=\dot{Q}_{-}$that measures the distortion of the curvature encodes all the information on a given odd perturbation, it is natural to define the strength of the perturbation as the magnitude of this field. A key additional feature of $\dot{Q}_{-}$is the fact $r^{5} \dot{Q}_{-}$satisfies equation (4DRWE) (see (105) in Theorem 1.(i)). This will be used to prove that the magnitude of $\dot{Q}_{-}$, and thus the strength of the perturbation, can be bounded on the entire outer static region. This fulfills the requirements of our proposed notion of non-modal linear stability. It is important to note that Theorem 1 applies to arbitrary smooth perturbations, whereas any boundedness or fall-off condition will require a restriction to perturbations evolving from data that behave properly as $r \rightarrow \infty\left(r \rightarrow r_{c}\right)$ in the asymptotically flat (de Sitter) case.

\section{The asymptotically flat case}

In the $\Lambda=0$ case, a simple pointwise boundedness statement for $G_{-}=\dot{Q}_{-}$can be made by noting that the proof of boundedness of a Klein Gordon field in [21] holds for the 4DRW equation:

Theorem 2. For any smooth solution of the odd LEE which has compact support on Cauchy surfaces of the Kruskal extension I $\cup \mathrm{II} \cup \mathrm{I}^{\prime} \cup \mathrm{II}^{\prime}$ of the Schwarzschild space-time (Figure 1), there exists a constant $K_{-}$such that $\left|G_{-}\right|<K_{-} r^{-6}$ for $r>2 M$.

Proof. From (104), $\left|\dot{Q}_{-}\right| \leq C / r^{7}+\left|r^{5} \dot{Q}_{>1}\right| / r^{5}$, where $C>0$ is a constant that depends on the $j^{(m)}$ 's and the field $r^{5} \dot{Q}_{>1}$ satisfies the 4DRW equation (100), so we only need concentrate on this field. The similarities between the 4DRWE equation and the massive Klein Gordon equation dealt with in [21] allow us to proceed by adapting the proof of Theorem 1 in [21]. The $Z_{2}$ symmetry arguments in [21] showing that this theorem reduces to the intermediate case (treated in the appendix in [21]) apply verbatim to equation (100). The intermediate case is defined by adding the requirements that $r^{5} \dot{Q}$ and its $T$ derivative (in coordinates $(T, X, \theta, \phi)$, where $T=(U+V) / 2$ and $X=(V-U) / 2$ ) vanish on the bifurcation sphere (refer to Section II B 2). There remains to check that the proof in the appendix in [21] applies to the wave equation (100). To this end, we use (65)-(68) to cast (105) in the exterior Kruskal wedge as

$$
\frac{\partial^{2}}{\partial t^{2}}\left(r^{6} \dot{Q}_{>1}\right)+\mathcal{A}\left(r^{6} \dot{Q}_{>1}\right)=0
$$

where

$$
\mathcal{A}=-\frac{\partial^{2}}{\partial r^{* 2}}+\left(1-\frac{2 M}{r}\right)\left(-\frac{6 M}{r^{3}}-\frac{\widehat{D}^{A} \widehat{D}_{A}}{r^{2}}\right)=:-\frac{\partial^{2}}{\partial r^{* 2}}+V_{1}-V_{2} \widehat{D}^{A} \widehat{D}_{A},
$$

with $r^{*}$ defined in (48),

$$
V_{1}=\left(1-\frac{2 M}{r}\right)\left(-\frac{6 M}{r^{3}}\right) \text { and } \quad V_{2}=\left(1-\frac{2 M}{r}\right)\left(\frac{1}{r^{2}}\right)
$$

For the Klein Gordon equation dealt with in [21], the differential equation assumes this same form with

$$
V_{1}^{K G}=\left(1-\frac{2 M}{r}\right)\left(\frac{2 M}{r^{3}}+m^{2}\right), \quad V_{2}^{K G}=V_{2},
$$

where $m^{2}$ is the square of the mass of the Klein Gordon field (which appears with a wrong sign in equation (1) in [21], but with the correct sign in the equations in the appendix). However, the proof in the appendix in [21] does not make use of the explicit forms of $V_{1}^{K G}$ and $V_{2}^{K G}$, but only on the facts that these functions are bounded on the exterior wedge $r \geq 2 M$, and that $\mathcal{A}$ is a positive definite self adjoint operator on $L^{2}\left(\mathbb{R} \times S^{2}, d r^{*} \sin \theta d \theta d \phi\right)$. Since $V_{1}$ and $V_{2}$ defined in (110)-(111) are bounded for $r>2 M$, and $\mathcal{A}$ is positive definite on the $\ell>1$ subspace of $L^{2}\left(\mathbb{R} \times S^{2}, d x \sin \theta d \theta d \phi\right)$, the proof in the appendix in [21] applies to the wave equation (105) for $r^{5} \dot{Q}_{-}^{>1}$. The intermediate case then follows, and so does the analogue of Theorem 1 in [21]. Note that the proof in [21] shows that for the Klein Gordon field $\left|\Phi_{K G}\right|<C / r$ holds on the domain of outer communications. Although the weaker statement $\left|\Phi_{K G}\right|<C$ has been made in [21], the stronger form was used in this proof. 


\section{The asymptotically de Sitter case}

Two key similarities between the extension (59) for $\Lambda \neq 0$ and the Kruskal extension of the $\Lambda=0$ Schwarzschild black hole (refer to Section II B 2 and Figure 1) are: i) the $Z_{2}$ isometry exchanging I and I' and II and II', and ii) the fact that $r_{h}$ is a simple root of $f$, which implies that the asymptotic behavior of fields vanishing at the bifurcation sphere is that in equation (A1) in [21]. This allows us to prove the following

Theorem 3. For any smooth solution of the odd LEE which has compact support on Cauchy surfaces of the extended $\mathrm{I} \cup \mathrm{II} \cup \mathrm{I}^{\prime} \cup \mathrm{II}^{\prime} S d S$ black hole, there exists a constant $K_{-}$such that $\left|G_{-}\right|<K_{-} r^{-6}$ (equivalently, $\left|G_{-}\right|<$a constant) for $r_{h}<r<r_{c}$.

Proof. As in the proof of Theorem 2, and in view of the above comments, we need only prove the intermediate case for $r^{5} \dot{Q}_{>1}$. This field obeys the equation

$$
\frac{\partial^{2}}{\partial t^{2}}\left(r^{6} \dot{Q}_{>1}\right)+\mathcal{A}^{\Lambda}\left(r^{6} \dot{Q}_{>1}\right)=0
$$

where (see (67))

$$
\mathcal{A}^{\Lambda}=-\frac{\partial^{2}}{\partial r^{* 2}}+\left(1-\frac{2 M}{r}-\frac{2 \Lambda}{3} r^{2}\right)\left(-\frac{6 M}{r^{3}}-\frac{\widehat{D}^{A} \widehat{D}_{A}}{r^{2}}\right)=:-\frac{\partial^{2}}{\partial r^{* 2}}+V_{1}^{\Lambda}-V_{2}^{\Lambda} \widehat{D}^{A} \widehat{D}_{A},
$$

with $-\infty<r^{*}<\infty$,

$$
V_{1}^{\Lambda}=\left(1-\frac{2 M}{r}-\frac{2 \Lambda}{3} r^{2}\right)\left(-\frac{6 M}{r^{3}}\right) \quad \text { and } \quad V_{2}^{\Lambda}=\left(1-\frac{2 M}{r}-\frac{2 \Lambda}{3} r^{2}\right)\left(\frac{1}{r^{2}}\right) .
$$

In view of (53) and the condition $\ell \geq 2, V_{1}^{\Lambda}$ and $V_{2}^{\Lambda}$ are bounded in region II $\left(r_{h}<r<r_{c}\right)$ and $V_{1}^{\Lambda}-V_{2}^{\Lambda} \widehat{D}^{A} \widehat{D}_{A}>0$, thus $\mathcal{A}^{\Lambda}$ is a positive definite self adjoint operator on $L^{2}\left(\mathbb{R} \times S^{2}, d r^{*} \sin \theta d \theta d \phi\right)$, and the proof follows as in Theorem 2 .

\section{A comment on the asymptotically anti de Sitter case}

The stability proofs above use the facts that the operators (110) and (114) are self adjoint and positive definite in the region of interest. For negative cosmological constant, the operator acts as (114) but on functions defined for $-\infty<r^{*}<0$, which is the outer static region in this case. This operator is only formally self adjoint (i.e., if we ignore boundary terms when integrating by parts), we need to specify boundary conditions at $r^{*}=0$ to properly define a domain where the operator is self adjoint. There are different options, and the positivity or not of the resulting operator depends on the chosen boundary condition [33]. The stability for Dirichlet boundary conditions was established in [13].

\section{THE LINEARIZED EINSTEIN EQUATION: EVEN SECTOR}

\section{A. Gauge transformations and gauge invariants}

The effect of the gauge transformation (70)-(72) in the even sector (36) is

$h_{\alpha \beta}^{\prime(+)}=\left(\begin{array}{cc}h_{a b}+\widetilde{D}_{a} \xi_{b}+\widetilde{D}_{b} \xi_{a} & \widehat{D}_{B}\left(q_{a}+r^{2} \widetilde{D}_{a} X+\xi_{a}^{>0}\right) \\ \widehat{D}_{A}\left(q_{b}+r^{2} \widetilde{D}_{b} X+\xi_{b}^{>0}\right) & r^{2}\left[\frac{1}{2}\left(J+2 \widehat{D}^{C} \widehat{D}_{C} X+\frac{4}{r} \xi^{a} \widetilde{D}_{a} r\right) \widehat{g}_{A B}+\left(2 \widehat{D}_{A} \widehat{D}_{B}-\widehat{g}_{A B} \widehat{D}^{C} \widehat{D}_{C}\right)(G+X>1)\right]\end{array}\right)$.

Since $X$ and $q_{a}$ belong to $L^{2}\left(S^{2}\right)_{>0}$, and $G \in L^{2}\left(S^{2}\right)_{>1}$, the $\ell=0,1$ modes require a separate treatment. 


$$
\text { 1. } \ell=0 \text { mode }
$$

For the $\ell=0$ mode

$$
h_{\alpha \beta}^{\prime(\ell=0,+)}=\left(\begin{array}{cc}
h_{a b}^{(\ell=0)}+\widetilde{D}_{a} \xi_{b}^{(\ell=0)}+\widetilde{D}_{b} \xi_{a}^{(\ell=0)} & 0 \\
0 & \widehat{g}_{A B}\left(J^{(\ell=0)}+\frac{4}{r} \xi_{(\ell=0)}^{a} \widetilde{D}_{a} r\right) \frac{r^{2}}{2}
\end{array}\right)
$$

we partially fix a gauge by requiring $J^{\prime(\ell=0)}=0$ together with the transverse condition $\tilde{g}^{a b} h^{\prime(\ell=0)}=0[27]($ note that a further gauge transformation with a gauge field

$$
\xi_{a}^{(\ell=0)}=\tilde{\epsilon}_{a b} \widetilde{D}^{b} Z(r)
$$

would preserve these two conditions.) Dropping the primes, the perturbation in such a traceless gauge reads

$$
h_{\alpha \beta}^{(\ell=0,+, T)}=\left(\begin{array}{cc}
h_{a b}^{(\ell=0, T)} & 0 \\
0 & 0
\end{array}\right), \quad \tilde{g}^{a b} h_{a b}^{(\ell=0, T)}=0 .
$$

For the traceless symmetric orbit space tensor $h_{a b}^{(\ell=0, T)}$ we use the identity [27]

$$
h_{a b}^{(\ell=0, T)}=\frac{1}{f}\left[C_{a}^{(\ell=0)} \widetilde{D}_{b} r+C_{b}^{(\ell=0)} \widetilde{D}_{a} r-\tilde{g}_{a b} C_{d}^{(\ell=0)} \widetilde{D}^{d} r\right], \quad C_{a}^{(\ell=0)}=h_{a b}^{(\ell=0, T)} \widetilde{D}^{b} r
$$

in terms of which, the residual gauge transformation along the field (118) gives

$$
C_{a}^{(\ell=0)} \rightarrow C_{a}^{\prime(\ell=0)}=C_{a}^{(\ell=0)}+f Z^{\prime \prime}(r) \tilde{\epsilon}_{a b} \widetilde{D}^{b} r
$$

In conclusion, we assume the form (119)-(120) for the metric perturbation, where $C_{a}^{(\ell=0)}$ is equivalent to $C^{\prime(\ell=0)}$ defined in (121).

$$
\text { 2. } \ell=1 \text { modes }
$$

For $\ell=1$ modes

$$
h_{\alpha \beta}^{(\ell=1,+)}=\left(\begin{array}{cc}
h_{a b}^{(\ell=1)}+\widetilde{D}_{a} \xi_{b}^{(\ell=1)}+\widetilde{D}_{b} \xi_{a}^{(\ell=1)} & \widehat{D}_{B}\left(q_{a}^{(\ell=1)}+r^{2} \widetilde{D}_{a} X^{(\ell=1)}+\xi_{a}^{(\ell=1)}\right) \\
\widehat{D}_{A}\left(q_{b}^{(\ell=1)}+r^{2} \widetilde{D}_{b} X^{(\ell=1)}+\xi_{b}^{(\ell=1)}\right) & \widehat{g}_{A B}\left(J^{(\ell=1)}-4 X^{(\ell=1)}+\frac{4}{r} \xi_{(\ell=1)}^{a} \widetilde{D}_{a} r\right) \frac{r^{2}}{2}
\end{array}\right)
$$

We may choose the gauge field such that $r^{2} \widetilde{D}_{a} X^{(\ell=1)}+\xi_{a}^{(\ell=1)}=-q_{a}^{(\ell=1)}$ and $2 \widetilde{D}^{b} \xi_{b}^{(\ell=1)}=-\tilde{g}^{a b} h_{a b}^{(\ell=1)}$. This will partially fix an orbit space transverse gauge, leaving a perturbation of the form

$$
h_{\alpha \beta}^{(\ell=1,+, T)}=\left(\begin{array}{cc}
h_{a b}^{(\ell=1, T)} & 0 \\
0 & \frac{r^{2}}{2} \widehat{g}_{A B} J^{(\ell=1)}
\end{array}\right), \quad \tilde{g}^{a b} h_{a b}^{(\ell=1, T)}=0 .
$$

where, as in the $\ell=0$ sector,

$$
h_{a b}^{(\ell=1, T)}=\frac{1}{f}\left[C_{a}^{(\ell=1)} \widetilde{D}_{b} r+C_{b}^{(\ell=1)} \widetilde{D}_{a} r-\tilde{g}_{a b} C_{d}^{(\ell=1)} \widetilde{D}^{d} r\right], \quad C_{a}^{(\ell=1)}=h_{a b}^{(\ell=1, T)} \widetilde{D}^{b} r
$$

A residual gauge transformation along a field (29) satisfying

$$
r^{2} \widetilde{D}_{a} X^{(\ell=1)}+\xi_{a}^{(\ell=1)}=0, \quad \widetilde{D}^{a} \xi_{a}^{(\ell=1)}=0
$$

preserves the form (123)-(124). 


$$
\text { 3. } \ell>1 \text { modes }
$$

For $\ell>1$, equation (116)

$$
\begin{aligned}
h_{a b}^{\prime>1} & =h_{a b}^{>1}+\widetilde{D}_{a} \xi_{b}^{>1}+\widetilde{D}_{b} \xi_{a}^{>1} \\
q_{a}^{\prime>1} & =q_{a}^{>1}+r^{2} \widetilde{D}_{a} X^{>1}+\xi_{a}^{>1} \\
J^{\prime>1} & =J+2 \widehat{D}^{C} \widehat{D}_{C} X^{>1}+\frac{4}{r} \xi_{a}^{>1} \widetilde{D}^{a} r \\
G^{\prime} & =G+X^{>1},
\end{aligned}
$$

has two implications (compare with the discussion following (74)): i) the fields

$$
\begin{aligned}
H_{a b} & =h_{a b}^{\ell>1}-\widetilde{D}_{a} p_{b}-\widetilde{D}_{b} p_{a}, \quad\left(p_{a} \equiv q_{a}^{>1}-r^{2} \widetilde{D}_{a} G\right) \\
\mathcal{J} & =J-\frac{4}{r} p_{a} \widetilde{D}^{a} r-2 \widehat{D}^{C} \widehat{D}_{C} G
\end{aligned}
$$

are gauge invariants; and (ii) there is a (unique) gauge (the Regge-Wheeler gauge) where the $\ell>1$ piece of the even metric perturbation assumes the form

$$
h_{\alpha \beta}^{(+,>1)}=\left(\begin{array}{cc}
H_{a b} & 0 \\
0 & \frac{r^{2}}{2} \widehat{g}_{A B} \mathcal{J}
\end{array}\right) .
$$

\section{B. The linearized Einstein equation}

\section{1. $\ell>1$ modes}

A calculation of the LEE (1) using (129) gives, in agreement with equations (31) in [5], the following components for $\dot{R}_{\alpha \beta}-\Lambda h_{\alpha \beta}$ :

$$
\begin{aligned}
\dot{R}_{a b}-\Lambda h_{a b}= & \frac{\widetilde{D}^{c} r}{r}\left(\widetilde{D}_{a} H_{b c}+\widetilde{D}_{b} H_{a c}-\widetilde{D}_{c} H_{a b}\right)-\frac{1}{2 r^{2}} \widehat{D}^{C} \widehat{D}_{C} H_{a b}+\frac{\tilde{R}}{2} H_{a b} \\
& +\frac{1}{2} \tilde{g}_{a b}\left(\widetilde{D}^{c} \widetilde{D}^{d} H_{c d}-\widetilde{D}^{c} \widetilde{D}_{c} H-\frac{\tilde{R}}{2} H\right)-\frac{1}{2 r^{2}} \widetilde{D}_{(a}\left(r^{2} \widetilde{D}_{b)} \mathcal{J}\right)-\Lambda H_{a b}, \\
\dot{R}_{a B}-\Lambda h_{a B}= & \frac{1}{2} \widehat{D}_{B}\left[\widetilde{D}^{b} H_{a b}-r \widetilde{D}_{a}\left(\frac{H}{r}\right)-\frac{1}{2} \widetilde{D}_{a} \mathcal{J}\right] \\
\dot{R}_{A B}-\Lambda h_{A B}= & -\frac{1}{2}\left(\widehat{D}_{A} \widehat{D}_{B} H-\frac{1}{2} \widehat{g}_{A B} \widehat{D}^{C} \widehat{D}_{C} H\right)+\widehat{g}_{A B}\left[\widetilde{D}^{a}\left(r H_{a b} \widetilde{D}^{b} r\right)\right. \\
& \left.-\frac{r}{2}\left(\widetilde{D}^{a} r\right) \widetilde{D}_{a} H-\frac{1}{4} \widetilde{D}^{c} \widetilde{D}_{c}\left(r^{2} \mathcal{J}\right)-\frac{1}{4} \widehat{D}^{C} \widehat{D}_{C}(H+\mathcal{J})-\Lambda \frac{r^{2}}{2} \mathcal{J}\right]
\end{aligned}
$$

where $\tilde{R}$ is the Ricci scalar of the orbit manifold and

$$
H=\tilde{g}^{a b} H_{a b}
$$

From part ii) of Lemma 2 (applied now to the symmetric tensor $\dot{R}_{\alpha \beta}-\Lambda h_{\alpha \beta}$ ) we conclude that (132) implies $H=0$, then as in (120) we introduce

$$
C_{a}^{>1}=H_{a b} \widetilde{D}^{b} r
$$

which gives

$$
H_{a b}=\frac{1}{f}\left[C_{a}^{>1} \widetilde{D}_{b} r+C_{b}^{>1} \widetilde{D}_{a} r-\tilde{g}_{a b} C_{d}^{>1} \widetilde{D}^{d} r\right]
$$


and

$$
\tilde{\epsilon}^{a c}\left(\widetilde{D}_{c} r\right) \widetilde{D}^{b} H_{a b}=\tilde{\epsilon}^{a c} \widetilde{D}_{c} C_{a} .
$$

For $\ell>0$, equation (131) is equivalent to $\widetilde{D}^{b} H_{a b}-\frac{1}{2} \widetilde{D}_{a} \mathcal{J}=0$. Contracting this equation with the orthogonal vectors $\widetilde{D}^{a} r$ and $\tilde{\epsilon}^{a b} \widetilde{D}_{b} r$ and using (91) gives [5]:

$$
\begin{aligned}
0 & =\widetilde{D}^{a} r\left(\widetilde{D}^{b} H_{a b}-\frac{1}{2} \widetilde{D}_{a} \mathcal{J}\right)=\widetilde{D}^{b}\left(H_{a b} \widetilde{D}^{a} r\right)-H_{a b} \widetilde{D}^{b} \widetilde{D}^{a} r-\frac{1}{2}\left(\widetilde{D}^{a} r\right)\left(\widetilde{D}_{a} \mathcal{J}\right) \\
& =\widetilde{D}^{b} C_{b}^{>1}-\frac{1}{2}\left(\widetilde{D}^{b} r\right)\left(\widetilde{D}_{b} \mathcal{J}\right) \\
0 & =\tilde{\epsilon}^{a c}\left(\widetilde{D}_{c} r\right)\left(\widetilde{D}^{b} H_{a b}-\frac{1}{2} \widetilde{D}_{a} \mathcal{J}\right) \\
& =\tilde{\epsilon}^{a c}\left(\widetilde{D}_{c} C_{a}^{>1}-\frac{1}{2}\left(\widetilde{D}_{c} r\right) \widetilde{D}_{a} \mathcal{J}\right) .
\end{aligned}
$$

Introducing

$$
Z_{a} \equiv C_{a}^{>1}-\frac{r}{2} \widetilde{D}_{a} \mathcal{J}
$$

we write (138) as

$$
\widetilde{D}_{[a} Z_{b]}=0
$$

and equation (137) as

$$
\widetilde{D}^{b} Z_{b}+\frac{r}{2} \widetilde{D}^{b} \widetilde{D}_{b} \mathcal{J}=0
$$

Now contract (132) with $\widehat{g}^{A B}$, this gives

$$
4 \widetilde{D}^{a}\left(r Z_{a}\right)+r^{2} \widetilde{D}^{c} \widetilde{D}_{c} \mathcal{J}-\left[\widehat{D}^{C} \widehat{D}_{C}+2\right] \mathcal{J}=0 .
$$

Finally, contracting the $\tilde{g}_{a b}$ trace-free part of (130) with $\widetilde{D}^{a} r$ and using (142) we arrive at

$$
\widetilde{D}_{a}\left[2 r\left(\widetilde{D}^{b} r\right) Z_{b}+(3 M-r) J-\frac{r}{2} \widehat{D}^{C} \widehat{D}_{C} J\right]-\widehat{D}^{C} \widehat{D}_{C} Z_{a}=0
$$

It is interesting to note that equations (140)-(143) look formally identical to the $\Lambda=0$ case, equations (41a)-(41d) in [5], $\Lambda$ appears only implicitly through $\tilde{g}_{a b}$ and its Levi-Civita derivative $\widetilde{D}_{a}$.

$$
\text { 2. } \ell=0 \text { mode }
$$

In deriving equations (130)-(132) we made no assumptions on $H_{a b}$ and $\mathcal{J}$ in (75), so these calculations apply to the $\ell=0$ mode (119) if we replace $H_{a b}$ with $h_{a b}^{(\ell=0, T)}$ and set $\mathcal{J}=0$. Equation (131) is void in this case, whereas equation (132) reduces to $\widetilde{D}^{a}\left(r C_{a}^{(\ell=0)}\right)=0$, whose solution is

$$
C_{a}^{(\ell=0)}=\frac{1}{r} \tilde{\epsilon}_{a b} \widetilde{D}^{b} z, \quad z: \mathcal{O} \rightarrow \mathbb{R} .
$$

In Schwarschild coordinates $(t, r)$ the residual gauge freedom (121) implies that $z(t, r)$ is defined up to an arbitrary additive function $x(r)$ (choose $x^{\prime}=r f Z^{\prime \prime}$ to match (121)). Replacing (144) in (130) and (132) we find that,working in $(t, r)$ coordinates, $z(t, r)=A t+B(r)$. This gives $h_{t t}=A / r, h_{r r}=(2 A / r) / f^{2}$ and $h_{t r}=B^{\prime}(r) / r$. Choosing the gauge $B(r)=0$ we recognize this perturbation as a shift $M \rightarrow M+A / 2$ in the mass treated to first order in $A / 2=\dot{M}$. This was to be expected from Birkhoff's theorem. In conclusion, we can choose a gauge such that

$$
C_{a}^{(\ell=0)}=\frac{2 \dot{M}}{r f} \widetilde{D}_{a} r
$$

The perturbation class is characterized by the parameter $\dot{M}$. 


\section{3. $\ell=1$ modes}

We use again equations (130)-(132) with the replacements $H_{a b} \rightarrow h_{a b}^{(\ell=1, T)}$ and $J \rightarrow J^{(\ell=1)}$, and find that the general solution to these equations can be set to zero using the residual gauge freedom (125) (see [27]). This implies that the even $\ell=1$ sector is void.

\section{Solution of the linearized Einstein equation}

The results of the previous section are gathered in the following

Lemma 5. The LEE (1) in the even sector is equivalent to the system of equations (139)-(143) and (145).

The system (140)-(143) of LEE for the $\ell>1$ sector was first solved by Zerilli in [32], the addition of a cosmological constant was considered in [17]. In this section we generalize to the case $\Lambda \neq 0$ the derivation in [5] of the Zerilli equation.

Equation (140) implies that there is a scalar field $\zeta: \mathcal{M} \rightarrow \mathbb{R}$, defined up to an additive function $z: S^{2} \rightarrow \mathbb{R}$, $\zeta=\zeta_{o}+z$, such that

$$
Z_{a}=\widetilde{D}_{a} \zeta
$$

From (141) and (142)

$$
\widetilde{D}^{b} Z_{b}+\frac{2 \widetilde{D}^{b} r}{r} Z_{b}-\frac{\left(\widehat{D}^{C} \widehat{D}_{C}+2\right)}{2 r} \mathcal{J}=0
$$

From (143) (146) and the above equation

$$
\widetilde{D}^{b} \widetilde{D}_{b} \zeta_{o}+\frac{\widehat{D}^{C} \widehat{D}_{C}}{r^{2}} \zeta_{o}-\frac{3 M}{r^{2}} \mathcal{J}=\frac{h-\widehat{D}^{C} \widehat{D}_{C} z}{r^{2}}, \quad h: S^{2} \rightarrow \mathbb{R}
$$

Since all fields above belong to $L^{2}\left(S^{2}\right)_{>1}$, we may choose $z$ such that the term on the right vanishes. From now on we assume this choice, which gives

$$
\widetilde{D}^{b} \widetilde{D}_{b} \zeta+\frac{\widehat{D}^{C} \widehat{D}_{C}}{r^{2}} \zeta-\frac{3 M}{r^{2}} \mathcal{J}=0
$$

Applying $\left(\widehat{D}^{C} \widehat{D}_{C}+2\right)$ to (149) and combining with (147) yields

$$
\left(\widehat{D}^{C} \widehat{D}_{C}+2-\frac{6 M}{r}\right) \widetilde{D}^{b} \widetilde{D}_{b} \zeta-\frac{12 M}{r^{2}} \widetilde{D}^{b} r \widetilde{D}_{b} \zeta+\frac{\left(\widehat{D}^{B} \widehat{D}_{B}\right)\left(\widehat{D}^{C} \widehat{D}_{C}+2\right)}{r^{2}} \zeta=0 .
$$

The Zerilli field

$$
\Psi=\left(\widehat{D}^{C} \widehat{D}_{C}+2-\frac{6 M}{r}\right)^{-1} \zeta
$$

is introduced to eliminate first derivatives from (150) and reduce it to a two dimensional wave equation:

$$
\begin{aligned}
\widetilde{D}^{b} \widetilde{D}_{b} \Psi+\frac{1}{r^{2}}\left(\widehat{D}^{B} \widehat{D}_{B}+2-\frac{6 M}{r}\right)^{-2}\left[\left(\widehat{D}^{B} \widehat{D}_{B}+2\right)^{2}\left(\widehat{D}^{B} \widehat{D}_{B}-\frac{6 M}{r}\right)\right. & \\
& \left.+\frac{36 M^{2}}{r^{2}}\left(\widehat{D}^{B} \widehat{D}_{B}+2-\frac{2 M}{r}+\frac{2}{3} \Lambda r^{2}\right)\right] \Psi=0 .
\end{aligned}
$$

This is the Zerilli equation, first obtained for $\Lambda=0$ in [32], and generalized to Schwarzschild-(A)dS in [17]. The non-local operator $\left(\widehat{D}^{B} \widehat{D}_{B}+2-6 M / r\right)^{-2}$ is well defined on $L^{2}\left(S^{2}\right)_{>1}$; the Zerilli equation was derived within the context of a modal approach to the problem, in which $\Psi$ is expanded in spherical harmonics

$$
\Psi=\sum_{(\ell, m)} \phi_{(\ell, m)}^{+} S_{(\ell, m)},
$$


and this operator reduces to $\left((\ell+2)(\ell-1)-\frac{6 M}{r}\right)^{-2}$ in the $\ell$ subspace, and it is therefore suitable to perform explicit calculations. If we use a tortoise radial coordinate $r^{*}$, we find that (152) is equivalent to the standard form (ZE)-(13) of the equation in the original references [32] [17]. From the $\zeta$ field (and therefore from the Zerilli field) it is possible to reconstruct $H_{a b}$ and $\mathcal{J}$ by tracing back the above equations. The result is [2] [5]:

$$
\begin{aligned}
J & =2\left(\widehat{D}^{C} \widehat{D}_{C}+2-\frac{6 M}{r}\right)^{-1}\left[2 \widetilde{D}^{b} r \widetilde{D}_{b} \zeta-\frac{1}{r} \widehat{D}^{B} \widehat{D}_{B} \zeta\right] \\
H_{a b} & =2\left(\widehat{D}^{C} \widehat{D}_{C}+2-\frac{6 M}{r}\right)^{-1}\left[\widetilde{D}_{a} \widetilde{D}_{b}(r \zeta)-\frac{\tilde{g}_{a b}}{2} \widetilde{D}^{c} \widetilde{D}_{c}(r \zeta)\right]
\end{aligned}
$$

The even sector LEE is equivalent to the set (150), (154)-(155).

\section{The ubiquitous Regge-Wheeler equation}

Let us consider the field [5]

$$
\Phi=-r \widetilde{D}^{b} \widetilde{D}_{b} \zeta
$$

Applying $\widetilde{D}^{a} \widetilde{D}_{a}$ to $r^{2}$ times equation (149), and eliminating $\widetilde{D}^{b} \widetilde{D}_{b} \mathcal{J}$ using (141), we find that $\Phi$ satisfies

$$
\widetilde{D}^{c} \widetilde{D}_{c} \Phi+\frac{2}{r} \widetilde{D}^{c} r \widetilde{D}_{c} \Phi+\frac{1}{r^{2}} \widehat{D}_{A} \widehat{D}^{A} \Phi+\left(\frac{6 M}{r^{3}}+\frac{1}{r} \widetilde{D}^{c} \widetilde{D}_{c} r\right) \Phi=0
$$

which is the Regge-Wheeler equation (100)! We have found a scalar field $\Phi$, related to the even metric perturbation potential $\zeta$ through (156), that satisfies the fundamental equation to which the odd LEE reduces.

Replacing $\widetilde{D}^{b} \widetilde{D}_{b} \zeta$ with $-\Phi / r$ in (150) gives the following relation between $\zeta$ and $\Phi$ :

$$
\left[12 M\left(\widetilde{D}^{a} r\right) \widetilde{D}_{a}-\left(\widehat{D}^{C} \widehat{D}_{C}+2\right)\left(\widehat{D}^{C} \widehat{D}_{C}\right)\right] \zeta=\left[\frac{6 M}{r}-\left(\widehat{D}^{C} \widehat{D}_{C}+2\right)\right](r \Phi) .
$$

Since $\zeta$ (or $\Psi$ ) contains all the information on equivalence classes of $\ell>1$ solutions of the even LEE, but does not admit a four dimensional translation, whereas $\Phi$ satisfies the 4DRW equation, one is tempted to treat even perturbations in terms of $\Phi$, using the relationship (158). This possibility was disregarded in [5] due to the fact that the operator on the left hand side in (158) has a non trivial kernel, suggesting that information is lost when switching from $\zeta$ to $\Phi$. There is, however, a loophole in this argument, as we now proceed to explain:

Expand $\Phi$ and $\zeta$ in spherical harmonics (we choose to call $\phi_{(\ell, m)}^{(-)}$the components of $\Phi$ in $(156)$ since this field satisfies (4DRWE), therefore the components (19) satisfy (RWE))

$$
\Phi=\sum_{\ell \geq 2, m} \frac{\phi_{(\ell, m)}^{(-)}}{r} S_{(\ell, m)}, \quad \zeta=\sum_{\ell \geq 2, m} \zeta_{(\ell, m)} S_{(\ell, m)}=-\sum_{\ell \geq 2, m}\left[\left[(\ell+2)(\ell-1)+\frac{6 M}{r}\right] \phi_{(\ell, m)}^{+} S_{(\ell, m)}\right.
$$

In $(t, r)$ coordinates, equation (158) with the replacements (159) reads

$$
\left[f \frac{\partial}{\partial r}-w_{\ell}\right] \zeta_{(\ell, m)}=\left[\frac{1}{2 r}+\frac{(\ell+2)(\ell-1)}{12 M}\right] \phi_{(\ell, m)}^{(-)},
$$

where

$$
w_{\ell}=\frac{1}{12 M} \frac{(\ell+2) !}{(\ell-2) !}
$$

are the frequencies of the Chandrasekhar algebraically special modes [3] [4]. The general solution of (160) can be written as

$$
\zeta_{(\ell, m)}(t, r)=F_{r_{o}}(t) e^{w_{\ell} r^{*}}-e^{w_{\ell} r^{*}} \int_{r^{*}}^{r_{o}^{*}} d r^{* \prime} e^{-w_{\ell} r^{* \prime}}\left[\phi_{(\ell, m)}^{(-)}(t, r)\left(\frac{1}{2 r\left(r^{* \prime}\right)}+\frac{(\ell+2)(\ell-1)}{12 M}\right)\right]_{r=r\left(r^{* \prime}\right)}
$$


where $r\left(r^{*}\right)$ is the inverse of the function $r^{*}(r)$ defined in (48), and $r_{o}$ is the radial coordinate of a point in the outer static region. The non-trivial kernel of the operator on the left of equation (158) is the reason why there is an arbitrary function of $t$ in the first term in (166). Note that

$$
F_{r_{o}}(t)=\zeta_{(\ell, m)}\left(t, r_{o}\right) e^{-w_{\ell} r_{o}^{*}}
$$

If we apply $\widetilde{D}^{b} \widetilde{D}_{b}$ to $\zeta_{(\ell, m)}$ in (162) and use (RWE) we find that (compare with equations (156) and (159))

$$
e^{-w_{\ell} r^{*}} f\left[\widetilde{D}^{b} \widetilde{D}_{b} \zeta_{(\ell, m)}+r^{-2} \phi_{(\ell, m)}^{(-)}\right]=\left(w_{\ell}^{2} F_{r_{o}}-\ddot{F}_{r_{o}}\right)+q_{r_{o}}(t),
$$

where

$$
\begin{aligned}
q_{r_{o}}(t)=\left[\left(\frac{w_{\ell}(\ell+2)(\ell-1)}{12 M}+\frac{w_{\ell}}{2 r_{o}}+\frac{1}{2 r_{o}^{2}}-\frac{M}{r_{o}^{3}}\right) \phi_{(\ell, m)}^{(-)}\left(t, r_{o}\right)\right. & \\
& \left.+\left(\frac{(\ell+2)(\ell-1)}{12 M}-\frac{\ell^{2}+\ell-5}{6 r_{o}}-\frac{M}{r_{o}^{2}}\right) \partial_{r} \phi_{(\ell, m)}^{(-)}\left(t, r_{o}\right)\right] e^{-w_{\ell} r_{o}^{*}}
\end{aligned}
$$

Let us consider equations (162)-(164) for different values of the cosmological constant:

- $\Lambda=0$ : in the asymptotically flat case (48) and (61) in (162) gives

$$
\begin{aligned}
& \zeta_{(\ell, m)}(t, r)=F_{r_{o}}(t) e^{w_{\ell} r}\left(\frac{r}{2 M}-1\right)^{2 M w_{\ell}} \\
& \quad-\left.\frac{e^{w_{\ell} r}}{2 M}\left(\frac{r}{2 M}-1\right)^{2 M w_{\ell}} \int_{r}^{r_{o}} r^{\prime} e^{-w_{\ell} r^{\prime}} \phi_{(\ell, m)}^{(-)}\right|_{\left(t, r^{\prime}\right)}\left(\frac{1}{2 r^{\prime}}+\frac{(\ell+2)(\ell-1)}{12 M}\right)\left(\frac{r^{\prime}}{2 M}-1\right)^{-2 M w_{\ell}-1} d r^{\prime},
\end{aligned}
$$

for some $r_{0}>2 M$.

According to Theorem 2, $\Phi$ in (158), being a solution of the $\Lambda=0$ (4DRWE), satisfies $|\Phi|<C / r$ on the exterior Kruskal wedge, then from $(159) \phi_{(\ell, m)}<$ constant [? ]. More generally, solutions of (RWE) behave either as $r^{\ell+1}$ or $r^{-\ell}$ for large $r$, and the first type should be discarded to preserve asymptotic flatness (alternatively, to assure the perturbative character of the initial datum). In any case, the integral in (166) converges if we take $r_{o}=\infty$ in $(166)$

$$
\begin{aligned}
& \zeta_{(\ell, m)}(t, r)=F_{\infty}(t) e^{w_{\ell} r}\left(\frac{r}{2 M}-1\right)^{2 M w_{\ell}} \\
& \quad-\left.\frac{e^{w_{\ell} r}}{2 M}\left(\frac{r}{2 M}-1\right)^{2 M w_{\ell}} \int_{r}^{\infty} r^{\prime} e^{-w_{\ell} r^{\prime}} \phi_{(\ell, m)}^{(-)}\right|_{\left(t, r^{\prime}\right)}\left(\frac{1}{2 r^{\prime}}+\frac{(\ell+2)(\ell-1)}{12 M}\right)\left(\frac{r^{\prime}}{2 M}-1\right)^{-2 M w_{\ell}-1} d r^{\prime},
\end{aligned}
$$

and the second term above is bounded for fixed $t$ as $r \rightarrow \infty$. Therefore, for metric perturbations that do not diverge as $r \rightarrow \infty$, it must be $F_{\infty}(t)=0$, otherwise the $\ell$ pieces of $J$ and $H_{a b}$ would behave for large $r$ as $e^{w_{\ell} r}$ times a rational function of $r$ (see (154)-(155)). We conclude that the asymptotic condition as $r \rightarrow \infty$ resolves the ambiguity in (158) and yields a 1-1 relation between the $\zeta$ and $\Phi$ fields:

$$
\zeta_{(\ell, m)}(t, r)=-\left.\frac{e^{w_{\ell} r}}{2 M}\left(\frac{r}{2 M}-1\right)^{2 M w_{\ell}} \int_{r}^{\infty} r^{\prime} e^{-w_{\ell} r^{\prime}} \phi_{(\ell, m)}^{(-)}\right|_{\left(t, r^{\prime}\right)}\left(\frac{1}{2 r^{\prime}}+\frac{(\ell+2)(\ell-1)}{12 M}\right)\left(\frac{r^{\prime}}{2 M}-1\right)^{-2 M w_{\ell}-1} d r^{\prime}
$$

Had we used $r_{o}<\infty$ in (166), the non-trivial function $F_{r_{o}}(t)$ could have been obtained by requiring that (166) be a solution of (156) (or, equivalently, of equation (150)) for $\phi_{(\ell, m)}$ satisfying (RWE). As follows from equation (164), this implies that $F_{r_{o}}(t)$ must be a solution of the ordinary differential equation resulting by setting the right hand side of this equation equal to zero. The ambiguity $A e^{w_{\ell} t}+B e^{-w_{\ell} t}$ in the solution of this equation is again fixed by adjusting $A$ and $B$ such that (162) remains bounded as $r \rightarrow \infty$ (this gives $A=B=0$ when $r_{o}=\infty$, in agreement with our previous paragraph).

Back to the case $r_{o}=\infty$, if we we let $F_{\infty} \neq 0$ in (167), allowing perturbations that diverge as $r \rightarrow \infty$, but require that

$$
\left(\partial_{t}^{2}-\partial_{r^{*}}^{2}\right)\left[F_{\infty}(t) e^{w_{\ell} r}\left(\frac{r}{2 M}-1\right)^{2 M w_{\ell}}\right]=\left(\partial_{t}^{2}-\partial_{r^{*}}^{2}\right)\left[F_{\infty}(t) \exp \left(w_{\ell} r^{*}\right)\right]=0
$$


to preserve (156), we obtain $\zeta_{\infty} \equiv F_{\infty}(t) \exp \left(w_{\ell} r^{*}\right)=\exp \left(w_{\ell}\left(r^{*} \pm t\right)\right)$ and, from (151), the following solutions to the Zerilli equation

$$
\phi_{(\ell, m)}^{(+)}=\frac{r \exp \left(w_{\ell}\left(r^{*} \pm t\right)\right)}{(\ell+2)(\ell-1) r+6 M} .
$$

The relevance of (170) (equivalently, the solutions $\zeta=\exp \left(w_{\ell}\left(r^{*} \pm t\right)\right)$ of $\left.(150)\right)$ comes from the fact that these are solutions of the Zerilli equation (respectively (150)) for any $\Lambda$ (assuming the appropriate $r^{*}(r)$ satisfying (48) is used) and, although useless when $\Lambda \geq 0$ for their behavior for large $r^{*}$, they are valid for $\Lambda<0$ since $r^{*}<0$ in this case, and show that there are unstable solutions in the asymptotically AdS case. This is discussed in detail in section IV E 2.

- $\Lambda>0$ : in this case $r_{o} \in\left(r_{h}, r_{c}\right)$ in (162). According to Theorem 3, since $\Phi$ in (158) is a solution of the $\Lambda>0$ (4DRWE), the $\phi_{(\ell, m)}$ in (160) are bounded for $r_{h}<r<r_{c}\left(-\infty<r^{*}<\infty\right)$. This implies that we can take $r_{o} \rightarrow r_{c}\left(r_{o}^{*} \rightarrow \infty\right)$ in (162), and the resulting term involving the integral will be bounded for fixed $t$ as $r \rightarrow r_{c}$. Therefore, as in the previous case, we conclude that for metric perturbations that do not diverge as $r \rightarrow r_{c}$, the only consistent choice is $F_{r_{c}}(t)=0$. Otherwise the $\ell$ pieces of $J$ and $H_{a b}$ would diverge as $e^{w_{e} r^{*}}$ as $r \rightarrow r_{c}$. Once again, the asymptotic condition resolves the ambiguity in (158) and yields a 1-1 relation between the $\zeta$ and $\Phi$ fields:

$$
\zeta_{(\ell, m)}(t, r)=-e^{w_{\ell} r^{*}} \int_{r^{*}}^{\infty} d r^{* \prime} e^{-w_{\ell} r^{* \prime}}\left[\phi_{(\ell, m)}^{(-)}(t, r)\left(\frac{1}{2 r}+\frac{(\ell+2)(\ell-1) r}{12 M}\right)\right]_{r=r\left(r^{* \prime}\right)}
$$

- $\Lambda<0$ : For asymptotically AdS black holes, we cannot use the argument above, since we have proven no analogue of Theorems 2 and 3 in this case. This is connected to the fact that there are different consistent choices for the behavior of the $\phi_{(\ell, m)}$ as $r \rightarrow \infty\left(r^{*} \rightarrow 0^{-}\right)$, and they lead to different dynamics [33]. As an example, a boundary condition consistent with (170) gives a dynamics under which the asymptotically AdS Schwarzschild black hole is unstable. The possibility of replacing the Zerilli equation (152) for the Regge-Wheeler equation (157) depends on the choice of boundary conditions at $r^{*}=0$. This is further investigated in section IVE and in [33].

We summarize below the results of this section:

Lemma 6. Consider the even sector of the LEE around a Schwarzschild-(A)dS background:

(i) The solution of the $\ell=0$ piece of the metric perturbation in a particular gauge is given by equations (119), (120) and (145).

(ii) The $\ell=1$ sector of the LEE is trivial.

(iii) For $\Lambda \geq 0$, the solution of the $\ell>1$ sector is given in equations (100), (159), (168)/(171) and (154)-(155).

(iv) For $\Lambda \geq 0$ there is a bijection between the space $\mathcal{L}_{+}$of smooth odd solutions of the LEE mod gauge transformations, and the set

$$
L_{+}=\{\dot{M}\} \cup\{\Phi \mid \Phi \text { is a smooth solution of equation (100) }\} .
$$

(v) For $\Lambda \geq 0$ there is a bijection between $\mathcal{L}_{+}$and the set

$$
L_{+}^{\phi}=\{\dot{M}\} \cup\left\{\phi_{(\ell, m)}^{+}, m, \ell \geq 2 \mid \phi_{(\ell, m)}^{+} \text {is a smooth solution of equation (ZE) }\right\} .
$$

We gather Lemma 4.iii and Lemma 6.iv in

Theorem 4. For $\Lambda \geq 0$ there is a bijection between the space $\mathcal{L}$ of smooth solutions of the LEE mod gauge transformations and the set

$$
L=\left\{\dot{M}, j^{(m)}, m=1,2,3\right\} \cup\left\{\left(\Phi_{-}, \Phi_{+}\right) \mid \Phi_{ \pm} \text {smooth solutions of equation (100) }\right\} .
$$

The dynamical perturbations are parametrized by the two solutions $\Phi_{ \pm}$of the $4 D R W$ equation (100), and correspond to $\ell \geq 2$ perturbations. The stationary perturbations are parametrized by the first order variation of the mass $\dot{M}$ $(\ell=0)$ and the angular momenta components $j^{(m)}, m=1,2,3(\ell=1)$, these correspond to perturbations within the Kerr/Kerr-(A)dS family. 


\section{E. Chandrasekhar's duality}

The modal approach to the linear perturbation problem is based on analyzing the evolution of isolated $(\ell, m)$ modes using the Zerilli and Regge-Wheeler equations (ZE) and (RWE) respectively. In $(t, r)$ coordinates, these equations are separable, there are solutions of the form

$$
\phi_{(\ell, m)}^{+}=\Re e^{i \omega t} \psi_{(\ell, m)}^{+}(r), \quad \phi_{(\ell, m)}^{-}=\Re e^{i \omega t} \psi_{(\ell, m)}^{-}(r),
$$

where $\psi_{(\ell, m)}^{ \pm}$satisfies a Schrödinger-like equation (note that $\mathcal{H}_{\ell}^{-}$agrees with the operator $\mathcal{A}^{\Lambda}$ introduced in $(67)$ )

$$
\mathcal{H}_{\ell}^{ \pm} \psi_{(\ell, m)}^{ \pm}:=\left[-\partial_{r^{*}}^{2}+U_{\ell}^{ \pm}\right] \psi_{(\ell, m)}^{ \pm}=\omega^{2} \psi_{(\ell, m)}^{ \pm},
$$

with potentials (see (12) (13))

$$
U_{\ell}^{+}=f V_{\ell}^{Z}, \quad U_{\ell}^{-}=f V_{\ell}^{R W}
$$

It was noticed by Chandrasekhar [3] [4] (see also Appendix A in [34]) that the $\mathcal{H}_{\ell}^{ \pm}=-\partial_{r^{*}}^{2}+U_{\ell}^{ \pm}$satisfy

$$
\mathcal{H}_{\ell}^{ \pm}+w_{\ell}^{2}=\mathcal{D}^{ \pm} \mathcal{D}^{\mp}
$$

where, generalizing Chandrasekhar's equations to $\Lambda \neq 0$,

$$
\begin{aligned}
& \mathcal{D}_{\ell}^{ \pm}= \pm \frac{\partial}{\partial r^{*}}+W_{\ell}, \\
& W_{\ell}=w_{\ell}+\frac{6 M f}{r(\mu r+6 M)},
\end{aligned}
$$

and $w_{\ell}$ are the frequencies (161). A consequence of the factorization (176) is that

$$
\begin{aligned}
& \mathcal{H}_{\ell}^{-} \psi_{(\ell, m)}^{-}=\omega^{2} \psi_{(\ell, m)}^{-} \Rightarrow \mathcal{H}_{\ell}^{+}\left(\mathcal{D}_{\ell}^{+} \psi_{(\ell, m)}^{-}\right)=\omega^{2}\left(\mathcal{D}_{\ell}^{+} \psi_{(\ell, m)}^{-}\right) \\
& \mathcal{H}_{\ell}^{+} \psi_{(\ell, m)}^{+}=\omega^{2} \psi_{(\ell, m)}^{+} \Rightarrow \mathcal{H}_{\ell}^{-}\left(\mathcal{D}_{\ell}^{-} \psi_{(\ell, m)}^{+}\right)=\omega^{2}\left(\mathcal{D}_{\ell}^{-} \psi_{(\ell, m)}^{+}\right)
\end{aligned}
$$

Similarly, solutions of the (RWE) and (ZE) are exchanged by $\mathcal{D}_{\ell}^{ \pm}$, e.g,

$$
\begin{aligned}
& \left(\partial_{t}^{2}-\partial_{r^{*}}^{2}+f V_{(\ell, m)}^{R W}\right) \phi_{(\ell, m)}^{-}=0 \Rightarrow\left(\partial_{t}^{2}-\partial_{r^{*}}^{2}+f V_{(\ell, m)}^{Z}\right)\left(\mathcal{D}_{\ell}^{+} \phi_{(\ell, m)}^{-}\right)=0 \\
& \left(\partial_{t}^{2}-\partial_{r^{*}}^{2}+f V_{(\ell, m)}^{Z}\right) \phi_{(\ell, m)}^{+}=0 \Rightarrow\left(\partial_{t}^{2}-\partial_{r^{*}}^{2}+f V_{(\ell, m)}^{R W}\right)\left(\mathcal{D}_{\ell}^{-} \phi_{(\ell, m)}^{+}\right)=0 .
\end{aligned}
$$

Chandrasekhar noticed the factorization (176) by casting the RW and Zerilli potentials in Riccati form, and finding the unexpected symmetry $U_{\ell}^{ \pm}= \pm \partial_{r^{*}} W_{\ell}+W_{\ell}^{2}-w_{\ell}^{2}$. We can trace origin of this symmetry to the previous to last equation in Section $\mathrm{V}$ of [5], which is equivalent to our equation (158) which, combined with (151) gives the $\mathcal{D}_{\ell}^{-}$ operator in (177)

\section{Case $\Lambda \geq 0$}

For $\Lambda \geq 0$, we have $-\infty<r^{*}<\infty, U_{\ell}^{ \pm} \rightarrow 0$ as $\left|r^{*}\right| \rightarrow \infty$, and we consider $\mathcal{H}_{\ell}^{ \pm}$as an operator in $L^{2}\left(\mathbb{R}_{r^{*}}, d r^{*}\right)$, where it is self-adjoint and positive. Since the general solution of the differential equation $\mathcal{D}_{\ell}^{-} \chi=0$ is a constant times

$$
\chi_{\ell}^{+}=\frac{r \exp \left(w_{\ell} r^{*}\right)}{(\ell+2)(\ell-1) r+6 M},
$$

and the general solution of the differential equation $\mathcal{D}_{\ell}^{+} \chi=0$ is a constant times

$$
\chi_{\ell}^{-}=\frac{(\ell+2)(\ell-1) r+6 M}{r} \exp \left(-w_{\ell} r^{*}\right)=\frac{1}{\chi_{\ell}^{+}},
$$

both $\mathcal{D}^{ \pm}$have trivial kernel in $L^{2}\left(\mathbb{R}_{r^{*}}, d r^{*}\right)$. Since the evolution of initial data $\left.\left(\phi_{(\ell, m)}^{ \pm}, \partial_{t} \phi_{(\ell, m)}^{ \pm}\right)\right|_{t_{o}}$ in $L^{2}\left(\mathbb{R}_{r^{*}}, d r^{*}\right)$ gives $\left.\phi_{(\ell, m)}^{ \pm}\right|_{t} \in L^{2}\left(\mathbb{R}_{r^{*}}, d r^{*}\right)$ for all $t$, we may consider, in view of (181) (182), replacing solutions of (ZE) with $\mathcal{D}_{\ell}^{-}$times solutions of (RWE). This was already shown to be possible for $\Lambda \geq 0$ in the previous section, equations (168) and (171). The duality involving the (ZE) and (RWE) is now reconsidered from the perspective offered by the factorization (176). 
Lemma 7. Assume $\Lambda \geq 0$. For any solution $\phi_{(\ell, m)}^{+}$of $(Z E)$ in $L^{2}\left(\mathbb{R}_{r^{*}}, d r^{*}\right)$ there is a unique solution $\phi_{(\ell, m)}^{-}$of $(R W E)$ in $L^{2}\left(\mathbb{R}_{r^{*}}, d r^{*}\right)$ such that

$$
\phi_{(\ell, m)}^{+}=\mathcal{D}_{\ell}^{+} \phi_{(\ell, m)}^{-} .
$$

The same statement holds switching + and - and (RWE) and (ZE).

Proof. We will only prove the first statement, as the proof of the second is completely analogous. Uniqueness follows from $\mathcal{D}_{\ell}^{-}$being injective. To prove existence, we use the fact, discovered by Price [25], that for $\Lambda=0, \phi_{(\ell, m)}^{+}$decays as $t^{-(2 \ell+2)}$ at large $t$ (the decay is exponential in $t$ if $\Lambda>0$ [39]) The time reversal symmetry of (ZE) indicates that this also happens for large negative $t$. This implies that $\phi_{(\ell, m)}^{+}$admits a Fourier representation

$$
\phi_{(\ell, m)}^{+}=\int_{-\infty}^{\infty} \widehat{\phi}_{(\ell, m)}^{+}\left(\omega, r^{*}\right) e^{i \omega t} d \omega .
$$

Note that

$$
\widetilde{\phi}_{(\ell, m)}^{+}=\int_{-\infty}^{\infty} \frac{\widehat{\phi}_{(\ell, m)}^{+}}{\omega^{2}+w_{\ell}^{2}} e^{i \omega t} d \omega .
$$

is also a solution of (ZE), therefore $\mathcal{D}_{\ell}^{-} \widetilde{\phi}_{(\ell, m)}^{+}$is a solution of (RWE). It is easy to show that this the solution sent to $\phi_{(\ell, m)}^{+}$by $\mathcal{D}_{\ell}^{+}$:

$$
\phi_{(\ell, m)}^{+}=\left(-\partial_{t}^{2}+w_{\ell}^{2}\right) \widetilde{\phi}_{(\ell, m)}^{+}=\left(\mathcal{H}_{\ell}^{+}+w_{\ell}^{2}\right) \widetilde{\phi}_{(\ell, m)}^{+}=\mathcal{D}_{\ell}^{+}\left(\mathcal{D}_{\ell}^{-} \widetilde{\phi}_{(\ell, m)}^{+}\right)
$$

\section{Case $\Lambda<0$ : instability of $S A d S$}

For SAdS, $-\infty<r^{*}<0$ (equation (62)) and, from (183), $\chi_{\ell}^{+} \in L^{2}\left(\mathbb{R}_{r^{*}}^{-}, d r^{*}\right)$ - the set of square integrable functions on the half line $r^{*}<0-$. Since $\mathcal{D}_{\ell}^{-} \chi_{\ell}^{+}=0$, we conclude that the operator $\mathcal{D}_{\ell}^{-}$fails to be injective in $L^{2}\left(\mathbb{R}_{r^{*}}^{-}, d r^{*}\right)$. Also, the potential or exponential decay of $\phi_{(\ell, m)}^{+}$for large $|t|$ at fixed position, required in the proof of Lemma 7 (equation (186)) allowing to replace solutions of (ZE) with solutions of (RWE) fails in this case.

In fact, using $\mathcal{D}_{\ell}^{-} \chi_{\ell}^{+}=0$ together with (174) we get the solution (170) of (ZE) found by Chandrasekhar in [3]. The choice of a plus sign in (170) gives

$$
\phi_{(\ell, m), \Lambda<0}^{+ \text {unst }}=\chi_{\ell}^{+}(r) \exp \left(w_{\ell} t\right)=\frac{r \exp \left(w_{\ell}\left(r^{*}+t\right)\right)}{(\ell+2)(\ell-1) r+6 M},
$$

which grows exponentially in time while remaining in $L^{2}\left(\mathbb{R}_{r^{*}}^{-}, d r^{*}\right)$ for every $t$, that is, is an unstable mode. In the RW gauge and using the coordinates $(v, r, \theta, \phi)$ in (47), the metric perturbation from (189) is given by a particularly simple expression (note that this is is well behaved across the horizon):

$$
h_{(\ell, m), \Lambda<0}^{+ \text {unst }}=\exp \left(w_{\ell} v\right) S_{(\ell, m)}\left[\frac{w_{\ell}}{6 M}(r \ell(\ell+1)-6 M) d v \otimes d v+\frac{\ell(\ell+1)}{6 M} r^{2}\left(d \theta \otimes d \theta+\sin ^{2}(\theta) d \phi \otimes d \phi\right)\right] .
$$

As shown in [36], generic perturbations of a Schwarzschild black hole give a type I spacetime, whereas the solution (189)-(190) splits one of the repeated principal null directions while preserving the degeneracy of the other one, leaving a type II spacetime.

As explained in Section II B 3, different dynamics are possible in SAdS depending on the boundary conditions imposed to the fields at the timelike boundary. Consider the Zerilli equation in the orbit space SAdS/SO(3)

$$
\left(\partial_{t}^{2}+\mathcal{H}_{\ell}^{+}\right) \phi_{(\ell, m)}^{+}=0 .
$$

The operator $\mathcal{H}_{\ell}^{+}$with domain the compactly supported functions on the half line $C_{o}^{\infty}\left(\mathbb{R}_{r^{*}}^{-}\right)$is symmetric, and it admits different self adjoint extensions within $L^{2}\left(\mathbb{R}_{r^{*}}^{-}, d r^{*}\right)$. Heuristically, self-adjoint extensions of operators like $\mathcal{H}_{\ell}^{+}$, 
which have a potential $U_{\ell}^{+}$that is regular at the boundary, are found by demanding that when integrating by parts the boundary terms do not spoil the transposition of the operator. As the following calculation shows,

$$
\begin{aligned}
\left(\psi_{2}, \mathcal{H}_{\ell}^{+} \psi_{1}\right) & =\int_{-\infty}^{0} \psi_{2}\left(-\partial_{r^{*}}^{2}+U_{\ell}^{+}\right) \psi_{1} d r^{*} \\
& =\left[\left(\partial_{r^{*}} \psi_{2}\right) \psi_{1}-\psi_{2}\left(\partial_{r^{*}} \psi_{1}\right)\right]_{r^{*}=-\infty}^{r^{*}=0}+\int_{-\infty}^{0} \psi_{1}\left(-\partial_{r^{*}}^{2}+U_{\ell}^{+}\right) \psi_{2} d r^{*} \\
& =\left[\left(\partial_{r^{*}} \psi_{2}\right) \psi_{1}-\psi_{2}\left(\partial_{r^{*}} \psi_{1}\right)\right]_{r^{*}=-\infty}^{r^{*}=0}+\left(\mathcal{H}_{\ell}^{+} \psi_{2}, \psi_{1}\right),
\end{aligned}
$$

this will be the case if we restrict $L^{2}\left(\mathbb{R}_{r^{*}}^{-}, d r^{*}\right)$ to the subspace $L_{\alpha}^{2}\left(\mathbb{R}_{r^{*}}^{-}, d r^{*}\right)$ of functions satisfying the boundary condition (see the discussion around equation (168) in [20], which applies to our case)

$$
\left.\partial_{r^{*}} \psi\right|_{r^{*}=0}=\left.\tan (\alpha) \psi\right|_{r^{*}=0},
$$

$\alpha \in[-\pi, \pi]$, where $\alpha= \pm \pi$ (to be identified) is understood as the Dirichlet boundary condition $\left.\psi\right|_{r^{*}=0}=0, \alpha=0$ corresponds to the Neumann boundary condition $\left(\left.\partial_{r^{*}} \psi\right|_{r^{*}=0}=0\right)$ and the remaining cases to Robin boundary conditions. We will call ${ }^{\alpha} \mathcal{H}_{\ell}^{+}$the self adjoint extension of $\mathcal{H}_{\ell}^{+}$to the domain $L_{\alpha}^{2}\left(\mathbb{R}_{r^{*}}^{-}, d r^{*}\right)$. Once a self-adjoint extension $\alpha$ is chosen, the dynamics is given by the curve in $L_{\alpha}^{2}\left(\mathbb{R}_{r^{*}}^{-}, d r^{*}\right)$ obtained by solving the equation [31]

$$
\partial_{t}^{2} \phi+{ }^{\alpha} \mathcal{H}_{\ell}^{+} \phi=0, \quad \phi \in L_{\alpha}^{2}\left(\mathbb{R}_{r^{*}}^{-}, d r^{*}\right),
$$

with initial conditions

$$
\left.\phi\right|_{t_{o}}=p,\left.\quad \partial_{t} \phi\right|_{t_{o}}=q, \quad p, q \in L_{\alpha}^{2}\left(\mathbb{R}_{r^{*}}^{-}, d r^{*}\right),
$$

and this is done by using the resolution of the identity for ${ }^{\alpha} \mathcal{H}_{\ell}^{+}$, i.e., by expanding in generalized eigenfunctions of this operator. If $E$ is a negative eigenvalue of ${ }^{\alpha} \mathcal{H}_{\ell}^{+}$, it belongs to the discrete part of the spectrum and, if $p_{E}$ and $q_{E}$ are the projections of $p$ and $q$ onto the $E$-eigenspace, the projection $\phi_{E}$ of $\phi$ in (194) will be

$$
\phi_{E}(t)=q_{E} \cosh (\sqrt{-E} t)+p_{E}(-E)^{-1 / 2} \sinh (\sqrt{-E} t) .
$$

Thus, there is an instability if the spectrum of ${ }^{\alpha} \mathcal{H}_{\ell}^{+}$contains negative eigenvalues, and whether this happens or not may depend on $\alpha$, so in general the issue of stability depends on what self adjoint extension ${ }^{\alpha} \mathcal{H}_{\ell}^{+}$(equivalently, what boundary condition at the timelike boundary) we choose to define the dynamics. As an example, in [37] Robin boundary conditions are enforced at a finite radius $r_{D}$ on the $\phi_{(\ell, m)}^{ \pm}$, as given in equations (2.32) and (2.42). This condition assures that there is a gauge for which the induced perturbed metric at the timelike hypersurface $r=r_{D}$ vanishes. One can check from the expressions in [37], however, that in the $r_{D} \rightarrow \infty$ limit (2.32) and (2.42) reduce to a the Dirichlet condition $\phi_{(\ell, m)}^{ \pm} / \partial_{r^{*}} \phi_{(\ell, m)}^{ \pm}=0$.

For the unstable mode $\chi_{\ell}^{+}$we find that it belongs to ${ }^{\alpha} \mathcal{H}_{\ell}^{+}$with

$$
\tan (\alpha)=\left.\frac{\partial_{r^{*}} \chi_{\ell}^{+}}{\chi_{\ell}^{+}}\right|_{r^{*}=0}=w_{\ell}-\frac{2 M \Lambda}{(\ell-1)(\ell+2)} .
$$

SAdS is unstable under dynamics that allow this boundary condition for some $\ell$. A systematic study of stability as a function of $\alpha$ is being carried out [33].

Note that the even/odd symmetry valid for $\Lambda \geq 0$ (equations (181)-(182)) and the related isospectrality of $\mathcal{H}_{\ell}^{+}$and $\mathcal{H}_{\ell}^{-}$(equations (179)-(180)) are in general broken in SAdS for generic boundary conditions [35] [38].

Note also the similarity between SAdS and the negative mass Schwarzschild solution, for which we may choose $r^{*}=0$ at $r=0$ and

$$
r^{*}=r+2 M \ln \left(1-\frac{r}{2 M}\right),
$$

will grow monotonically with $0<r^{*}<\infty$. The similarity with SAdS is that $r^{*}$ is also restricted to a half-line. This allows to consider the Chandrasekhar mode (the choice of a minus sign in (170))

$$
\phi_{(\ell, m), M<0}^{+ \text {unst }}=\chi_{\ell}^{+}(r) \exp \left(-w_{\ell} t\right)=\frac{r \exp \left(w_{\ell}\left(r^{*}-t\right)\right)}{(\ell+2)(\ell-1) r+6 M},
$$


as a possible solution of (ZE), as it belongs for every $t$ to the relevant space $L^{2}\left(\mathbb{R}_{r^{*}}^{+}, d r^{*}\right)$ (note that $w_{\ell}<0$ if $M<0$ ). This solution grows exponentially in time, so it signals an instability. There is, however, a key difference between the timelike boundary at $r=0$ of the negative mass Schwarzschild solution and the conformal timelike boundary at $r=\infty$ of SAdS, as for the former there is a unique boundary condition that makes the linear perturbation scheme self-consistent, by no worsening the degree of the pole of the unperturbed curvature scalars [16] [10]. The solution (199) of (ZE) satisfies precisely this boundary condition. This was used in [16] [10] to prove the instability of the Schwarzschild naked singularity.

\section{F. Measurable effects of the perturbation on the geometry}

In this section we show that there is a gauge invariant combination $G_{+}$of first order variation of the CSs (7) that contains all the gauge invariant information about the metric perturbation class $\left[h_{\alpha \beta}^{(+)}\right]$. In particular, $h_{\alpha \beta}^{(+)}$in the RW gauge can be obtained from $G_{+}$. Following [9], we introduce the differential curvature scalars (7). The background value of $X$ in the $\mathrm{S}(\mathrm{A}) \mathrm{dS}$ geometry is

$$
X_{S(A) d S}=\frac{M^{2}}{r^{9}}(r-2 M)-\frac{\Lambda M^{2}}{3 r^{6}},
$$

and the background value of $Q_{+}$in (7) is

$$
Q_{+S(A) d S}=\frac{M^{2}}{r^{6}}
$$

It follows that the combination

$$
G_{+}=\left(9 M-4 r+\Lambda r^{3}\right) \dot{Q}_{+}+3 r^{3} \dot{X}
$$

is gauge invariant since, under a gauge transformation along $\zeta^{\alpha}$,

$$
\begin{aligned}
G_{+} & \rightarrow G_{+}+\left(9 M-4 r+\Lambda r^{3}\right) £_{\zeta} Q_{+S(A) d S}+3 r^{3} £_{\zeta} X_{S(A) d S} \\
& =G_{+}+\left(9 M-4 r+\Lambda r^{3}\right) \zeta^{r} \partial_{r} Q_{+S(A) d S}+3 r^{3} \zeta^{r} \partial_{r} X_{S(A) d S} \\
& =G_{+}
\end{aligned}
$$

A lengthy calculation with the help of symbolic manipulation programs gives $G_{+}$for an arbitrary perturbation class $\left[h_{\alpha \beta}^{(+)}\right]$using the parametrization $L_{+}^{\phi}$ in Lemma 6.v and Schwarzschild coordinates $(t, r)$ :

$$
G_{+}=-\frac{2 M \dot{M}}{r^{5}}+\frac{M}{2 r^{4}} \sum_{\ell \geq 2} \frac{(\ell+2) !}{(\ell-2) !}\left[f \partial_{r}+Z_{\ell}\right] \phi_{(\ell, m)}^{+} S_{(\ell, m)},
$$

where

$$
Z_{\ell}=\frac{2 M \Lambda r^{3}+\mu r(r-3 M)-6 M^{2}}{r^{2}(\mu r+6 M)}, \quad \mu=(\ell-1)(\ell+2)
$$

This generalizes the result equation (33) in [9] to the case $\Lambda \neq 0$.

Theorem 5. Let $\left[h_{\alpha \beta}^{(+)}\right] \in \mathcal{L}_{+}$and $G_{+}\left(\left[h_{\alpha \beta}^{(+)}\right]\right)$be the field (204) for the perturbation class $\left[h_{\alpha \beta}^{(+)}\right]$. The map $\left[h_{\alpha \beta}^{(+)}\right] \rightarrow$ $G_{+}\left(\left[h_{\alpha \beta}^{(+)}\right]\right)$is invertible: it is possible to construct a representative of $\left[h_{\alpha \beta}^{(+)}\right]$from $G_{+}\left(\left[h_{\alpha \beta}^{(+)}\right]\right)$.

Proof. We will prove that the linear map $\left[h_{\alpha \beta}^{(+)}\right] \rightarrow G_{+}\left(\left[h_{\alpha \beta}^{(+)}\right]\right)$has trivial kernel. Assume that $\left(\dot{M}_{(1)},\left\{\phi_{(\ell, m)}^{+}{ }^{(1)}\right\}\right)$ and $\left(\dot{M}_{(2)},\left\{\phi_{(\ell, m)}^{+}{ }^{(2)}\right\}\right)$ give the same $G_{+}$, then expanding $G_{+}$in spherical harmonics we find that $\dot{M}_{(1)}=\dot{M}_{(2)}$ and also

$$
\left[f \partial_{r}+Z_{\ell}\right] \chi_{(\ell, m)}=0, \quad\left(\chi_{(\ell, m)}=\phi_{(\ell, m)}^{+}{ }^{(1)}-\phi_{(\ell, m)}^{+}{ }^{(2)}\right) .
$$


The general solution of equation (206) is

$$
\chi_{(\ell, m)}(t, r)=\frac{F^{(\ell, m)}(t)}{(6 M+\mu r)} \sqrt{1-\frac{2 M}{r}-\frac{\Lambda r^{3}}{3}} .
$$

Since $\chi_{(\ell, m)}(t, r)=\phi_{(\ell, m)}^{+}{ }^{(1)}-\phi_{(\ell, m)}^{+}{ }^{(2)}$, it must satisfy Zerilli's equation. Inserting (207) in (ZE) gives

$$
\frac{d^{2} F^{(\ell, m)}}{d t^{2}}-\left(\frac{(\mu+1) \Lambda}{3}-\frac{\mu}{r^{2}}+\frac{2 M(\mu-2)}{r^{3}}+\frac{9 M^{2}}{r^{4}}\right) F^{(\ell, m)}=0, \quad(\mu=(L-1)(L+2)),
$$

which only admits the trivial solution $F(t)=0$.

To construct a representative of $\left[h_{\alpha \beta}^{+}\right]$we need $\dot{M}$ and the $\phi_{(\ell, m)}^{+}$, together with equations $(119),(120),(145),(75)$, (151), (153), (154) and (155). Expanding $G_{+}$in spherical harmonics

$$
G_{+}=\sum_{(\ell, m)} G_{+}^{(\ell, m)} S_{(\ell, m)}
$$

we find that $\dot{M}=-r^{5} G_{+}^{(\ell=0)} /(2 M)$ and $\phi_{(\ell, m)}^{+}$is the only solution of

$$
\left[f \partial_{r}+Z_{\ell}\right] \phi_{(\ell, m)}^{+}=\frac{2 r^{4}}{M} \frac{(\ell-2) !}{(\ell+2) !} G_{+}^{(\ell, m)}
$$

that satisfies (ZE).

\section{G. Non-modal linear stability of the $\Lambda \geq 0$ black holes}

In this section we establish the pointwise boundedness of $G_{+}$, equation (204), on region II of a Schwarschild or SdS black hole. For $\Lambda<0$ and certain boundary conditions at the timelike boundary, this fails to be true. As an example, for boundary conditions allowing the unstable mode (189), $G_{+}$contains a contribution proportional to

$$
G_{+}\left(\left[h_{(\ell, m)}^{+ \text {unst }}\right]\right)=G_{(\ell, m)}^{+ \text {unst }}=\frac{M}{2 r^{4}} \frac{(\ell+2) !}{(\ell-2) !}\left(\frac{\ell(\ell+1)}{12 M}-\frac{1}{2 r}\right) \exp \left(w_{\ell} v\right) S_{(\ell, m)} .
$$

where $h_{(\ell, m)}^{+ \text {unst }}$ is given in $(190)$.

Back to the $\Lambda \geq 0$ case, we know from Lemma 7 that for a given a solution $\phi_{(\ell, m)}^{+}$of the (ZE), there exists a solution $\phi_{(\ell, m)}^{-}$of the (RWE) such that $\phi_{(\ell, m)}^{+}=\mathcal{D}^{+} \phi_{(\ell, m)}^{-}$. Using this replacement in (204) gives

$$
\begin{aligned}
{\left[f \partial_{r}+Z_{\ell}\right] \phi_{(\ell, m)}^{+}=\left[f \partial_{r}+Z_{\ell}\right]\left[f \partial_{r}+W_{\ell}\right] \phi_{(\ell, m)}^{-} } & \\
=\partial_{r^{*}}^{2} \phi_{(\ell, m)}^{-} & +\left(\partial_{r^{*}} W_{\ell}+Z_{\ell} W_{\ell}\right) \phi_{(\ell, m)}^{-}+\left(W_{\ell}+Z_{\ell}\right) \partial_{r^{*}} \phi_{(\ell, m)}^{-} \\
& =\partial_{t}^{2} \phi_{(\ell, m)}^{-}+\left(f V_{\ell}^{R W}+\partial_{r^{*}} W_{\ell}+Z_{\ell} W_{\ell}\right) \phi_{(\ell, m)}^{-}+\left(W_{\ell}+Z_{\ell}\right) \partial_{r^{*}} \phi_{(\ell, m)}^{-}
\end{aligned}
$$

Since

$$
f V_{\ell}^{R W}+\partial_{r^{*}} W_{\ell}+Z_{\ell} W_{\ell}=-\frac{1}{6} \Lambda \ell(\ell+1)+\frac{\Lambda M+w_{\ell}}{r}+\frac{\ell(\ell+1)-6 M w_{\ell}}{2 r^{2}}-\frac{(\ell(\ell+1)+3) M}{r^{3}}+\frac{6 M^{2}}{r^{4}}=: \sum_{j=0}^{4} P_{j}(\ell) r^{-j}
$$

and

$$
W_{\ell}+Z_{\ell}=w_{\ell}+\frac{r-3 M}{r^{2}}
$$

we can re write (204) as

$$
G_{+}=-\frac{2 M \dot{M}}{r^{5}}+\frac{M}{2 r^{3}}\left[\partial_{t}^{2} \Phi_{5}+\sum_{j=0}^{4} r^{-j} \Phi_{j}+\frac{f(r-3 M)}{r^{3}} \Phi_{5}+\frac{f}{r} \Phi_{6}\right]+\frac{M}{2 r^{3}} \partial_{r^{*}} \Phi_{6}+\frac{M(r-3 M)}{2 r^{5}} \partial_{r^{*}} \Phi_{5},
$$


where

$$
\begin{aligned}
\Phi_{j} & =\sum_{(\ell \geq 2, m)} \frac{(\ell+2) !}{(\ell-2) !} P_{j}(\ell) \frac{\phi_{(\ell, m)}}{r} S_{(\ell, m)}, j=0,1,2,3,4 \\
\Phi_{5} & =\sum_{(\ell \geq 2, m)} \frac{(\ell+2) !}{(\ell-2) !} \frac{\phi_{(\ell, m)}}{r} S_{(\ell, m)}, \\
\Phi_{6} & =\sum_{(\ell \geq 2, m)} \frac{(\ell+2) !}{(\ell-2) !} w_{\ell} \frac{\phi_{(\ell, m)}}{r} S_{(\ell, m)},
\end{aligned}
$$

are all solutions of (4DRWE), and therefore so is $\partial_{t}^{2} \Phi_{5}$. Note that the expression (215) for $G_{+}$, written entirely in terms of solutions of (4DRWE) is possible thanks to the cancellation in $(213)$ of the $((\ell+2)(\ell-1) r+6 M)$ denominators in $Z_{\ell}$ and $W_{\ell}$.

\section{Theorem 6 .}

(i) For any smooth solution of the even LEE which has compact support on Cauchy surfaces of the Kruskal extension $\mathrm{I} \cup \mathrm{II} \cup \mathrm{I}^{\prime} \cup \mathrm{II}^{\prime}$ of the Schwarzschild space-time, there exists a constant $K_{+}$such that $\left|G_{+}\right|<K_{+} r^{-4}$ for $r>2 M$.

(ii) For any mooth solution of the even LEE which has compact support on Cauchy surfaces of region I $\cup$ II $\cup \mathrm{I}^{\prime} \cup \mathrm{II}^{\prime}$ of the extended SdS black hole, there exists a constant $K_{+}$such that $\left|G_{+}\right|<K_{+} r^{-4}$ (equivalently $\left|G_{+}\right|<$some constant) for $r_{h}<r<r_{c}$.

Proof. We treat simultaneously the $\Lambda=0$ and $\Lambda>0$ cases. For even solutions of the LEE with compact support on Cauchy surfaces of the Kruskal extension, there is an open neighbourhood $\mathcal{N}$ of $r^{*}=\infty$ where $\phi_{(\ell, m)}^{+}=0$. This set is of the form $\mathcal{N}=\left\{\left(t, r^{*}, \theta, \phi\right) \mid r^{*}>R^{*}(t)\right\}$ where, for large $t, R^{*}(t)=t+$ constant, and there is a similar open neighbourhood $\mathcal{N}^{\prime}$ near the $\mathcal{I}^{+}$boundary of the isometric region $\mathrm{II}^{\prime}$.

In (212), we replaced the $\phi_{(\ell, m)}^{+}$fields with $\phi_{(\ell, m)}^{-}$fields according to (185). Using $\left.\phi_{(\ell, m)}^{+}\right|_{\mathcal{N}}=0$, equations $(177)-(178)$, (176) and (184), and the fact that $\phi_{(\ell, m)}^{-}$satisfies $(\mathrm{RWE})$, we find that, in $\mathcal{N}$,

$$
\phi_{(\ell, m)}^{-}=\frac{(\ell+2)(\ell-1) r+6 M}{r} \exp \left(-w_{\ell} r^{*}\right)\left[A_{(\ell, m)} \exp \left(-w_{\ell} t\right)+B_{(\ell, m)} \exp \left(w_{\ell} t\right)\right]
$$

According to Theorems 2 and 3, solutions $\Phi$ of the 4DRWE with compact support on Cauchy slices of the Kruskal extension satisfy $|\Phi|<C / r$ in region II. The 4 DRW fields $\Phi_{j}, j=0, \ldots, 6$ in equation (215) do not have compact support on Cauchy slices, their spherical harmonic components have the exponential tails (219). In what follows we prove that the results in Theorems 2 and 3 hold also in this case. This will allow us to place a pointwise bound to the term between square brackets in $G_{+}$, equation (215).

Let $\Phi$ be any of the $\Phi_{j}, j=0, \ldots, 6$, and write $\Phi$ as a sum of three solutions of the $4 \mathrm{DRWE}, \Phi=\Phi_{(a)}+\Phi_{(b)}+\Phi_{(c)}$, where $\Phi_{(a)}$ has compact support on Cauchy slices of the Kruskal extension and $\Phi_{(a)}=0$ for $r^{*}>R^{*}(t)+\epsilon$ for some positive $\epsilon, \Phi_{(b)}$ is supported in $r^{*} \in\left(R^{*}(t)-\epsilon, \infty\right)$ and $\Phi_{(c)}$ is similarly supported near the $\mathcal{I}^{+}$boundary of region $\mathrm{II}^{\prime}$ (this is done by writing the $\Phi$ datum on a $t$ slice as a sum of three appropriate terms and letting them evolve). In region II, $\Phi=\Phi_{(a)}+\Phi_{(b)}$ and $\Phi_{(a)}<C_{(a)} / r$ since its satisfies the hypothesis in Theorem 2 (3). On the other hand, $\Phi_{(b)}$ decays as in (219). Since this field is bounded away the bifurcation sphere, we can adapt the proof of boundedness in the Appendix in [21]. This proof follows two steps: i) the use of the Sobolev-type inequality

$$
|r \Phi|_{t} \mid \leq K\left(\|\Phi\|+\left\|r^{-1} \partial_{r^{*}}^{2}(r \Phi)\right\|+\left\|\mathbf{J}^{2} \Phi\right\|\right)
$$

where the norm

$$
\|\Phi\|=\langle\Phi \mid \Phi\rangle^{1 / 2}
$$

is given by the inner product

$$
\langle\Phi \mid \Psi\rangle=\int \Phi \Psi r^{2} d r^{*} \sin (\theta) d \theta d \phi,
$$

and ii) the replacement of each of the terms on the right hand side of (220) with $t$-independent quantities, which gives $|\Phi|<$ constant $/ r$. Since equation (220) holds for finite norm fields, we only need to prove that step ii) is feasible for $\Phi_{(b)}$. We will prove this avoiding the use of the operators $\mathcal{A}^{-1 / 2}$ and $\left.\left(\mathcal{A}^{\Lambda}\right)^{-1 / 2}\right)$ introduced in [21], applying instead 
the equivalent time integral technique introduced in sections 3.4 and 3.5 of [8] for the similar problem of proving the pointwise boundedness of $\Phi_{K G}$ on the static region of an extreme Reissner-Nordström black hole, $\Phi_{K G}$ a solution of the Klein-Gordon equation (note that $s$ is used in [8] for the tortoise coordinate $r^{*}$ ). The idea is the following: for solutions $\Phi \in L^{2}\left(S^{2}\right)_{>1}$ of the 4DRWE with finite norm there is a conserved (i.e., time independent) energy given by

$$
\mathcal{E}[\Phi]=\left\langle\partial_{t} \Phi \mid \partial_{t} \Phi\right\rangle+\left\langle\Phi \mid r^{-1} \mathcal{A}^{\Lambda}(r \Phi)\right\rangle
$$

This is easily checked using the form (66) of the 4DRWE. Note that both terms contributing to $\mathcal{E}[\Phi]$ are positive for a finite norm $\Phi \in L^{2}\left(S^{2}\right)_{>1}$, since $V_{1}-V_{2} \widehat{D}^{A} \widehat{D}_{A}>0$ as a consequence of (53) and (68) and $\partial_{r^{*}}^{2}>0$. Now assume there is a finite energy time integral $\tilde{\Phi}$ of $\Phi$, that is, $\tilde{\Phi}$ satisfies the 4DRWE and $\partial_{t} \tilde{\Phi}=\Phi$. Then $\mathbf{J}^{2} \tilde{\Phi}$ also satisfies the 4DRWE and

$$
\begin{aligned}
\|\Phi\|^{2} & \leq \mathcal{E}[\tilde{\Phi}] \\
\left\|r^{-1} \partial_{r^{*}}^{2}(r \Phi)\right\|^{2} & \leq \mathcal{E}\left[\partial_{t} \Phi\right] \\
\left\|\mathbf{J}^{2} \Phi\right\|^{2} & \leq \mathcal{E}\left[\mathbf{J}^{2} \tilde{\Phi}\right] .
\end{aligned}
$$

(compare with the set of equations above (A2) in [21], and with equation (76) in [8]). This allows us to replace the right hand side of equation (220) with a time independent constant, and get the desired bound $\Phi<C / r$. Note that (224) and (226) follow straightforwardly from the definition (223) and the comments below it, whereas (225) follows from $\mathcal{E}\left[\partial_{t} \Phi\right] \geq\left\|\partial_{t}^{2} \Phi\right\|^{2}=\left\|r^{-1} \mathcal{A}^{\Lambda}(r \Phi)\right\|^{2}>\left\|r^{-1} \partial_{r^{*}}^{2}(r \Phi)\right\|^{2}$. The existence of time integrals $\tilde{\Phi}_{(a)}$ of $\Phi_{(a)}$ and $\tilde{\Phi}_{(b)}$ of $\Phi_{(b)}$ can be proved following the steps in section 3.4 of [8], since these fields belong to the Hilbert space $\hat{\mathcal{H}}$ introduced in Lemma 2 of this reference (with the appropriate replacements of $V_{1}$ and $V_{2}$ ). To prove that $\tilde{\Phi}_{(a)}$ has finite energy we proceed as in Lemma 3 in [8], since $\Phi_{(a)}$ supported away of $r^{*}=\infty$. To prove that $\tilde{\Phi}_{(b)}$ has finite energy, we use (219) to show that, in $\mathcal{N}$

$$
\tilde{\phi}_{(b)(\ell, m)}=\frac{(\ell+2)(\ell-1) r+6 M}{w_{\ell} r} \exp \left(-w_{\ell} r^{*}\right)\left[-A_{(\ell, m)} \exp \left(-w_{\ell} t\right)+B_{(\ell, m)} \exp \left(w_{\ell} t\right)\right]+\psi_{(\ell, m)}^{-, o}(r)
$$

where $\psi_{(\ell, m)}^{-, o}(r)$ is a zero mode $(\omega=0)$ solution of equation $(174)$, that is $\mathcal{A} \psi_{(\ell, m)}^{-, o}=0\left(\mathcal{A}^{\Lambda} \psi_{(\ell, m)}^{-, o}=0\right)$. The asymptotic form for large $r^{*}$ for a zero mode is

$$
\psi_{(\ell, m)}^{-, o} \sim \begin{cases}A_{(\ell, m)}\left[r^{*-\ell}+\ldots\right]+B_{(\ell, m)}\left[r^{* \ell+1}+\ldots\right] & , \Lambda=0 \\ A_{(\ell, m)}\left[e^{-\alpha r^{*}}+\ldots\right]+B_{(\ell, m)}[1+\ldots] & , \Lambda>0\end{cases}
$$

where $\alpha>0$ is defined by equation (63) together with $e^{-\alpha r^{*}}=\left(r-r_{c}\right) / r_{c}$. For the unique time integral in $\hat{\mathcal{H}}$ of Lemma 2 in [8] it must be $B_{(\ell, m)}=0$ in (228). This implies that the energy integrals (224) and (226) converge for $\Phi=\tilde{\Phi}_{(b)}$. We conclude that $\Phi_{(b)}<C_{(b)} / r$ and that the sum of terms within square brackets in (215) is bounded by $C^{\prime} / r$ for some positive $C^{\prime}$.

To deal with the last two terms in (215) we need to prove the pointwise boundedness of $\partial_{r^{*}} \Phi$ in region II for $\Phi$ a solution of the 4DRW, $\Phi=\Phi_{(a)}+\Phi_{(b)}+\Phi_{(c)}$ as above. We do so by adapting the proof in section 3.6 in [8] of the pointwise boundedness of $\partial_{r^{*}}\left(r \Phi_{K G}\right)$. For $\Phi=\Phi_{(a)}$ or $\Phi=\Phi_{(b)}$ start from the Sobolev inequality (220) with $\Phi$ replaced with $r^{-1} \partial_{r^{*}}(r \Phi)$ :

$$
\left|\partial_{r^{*}}(r \Phi)\right|_{t} \mid \leq K\left(|| r^{-1} \partial_{r^{*}}(r \Phi)\|+\| r^{-1} \partial_{r^{*}}^{3}(r \Phi)\|+\| \mathbf{J}^{2} r^{-1} \partial_{r^{*}}(r \Phi) \|\right)
$$

The square of the first term on the right hand side is bounded by the time independent energy of $\Phi$ :

$$
\begin{aligned}
\mathcal{E}[\Phi] & =\left\langle\partial_{t} \Phi \mid \partial_{t} \Phi\right\rangle+\left\langle\Phi \mid r^{-1} \mathcal{A}^{\Lambda}(r \Phi)\right\rangle \\
& \geq\left\langle\Phi \mid r^{-1} \mathcal{A}^{\Lambda}(r \Phi)\right\rangle \\
& \geq\left\langle\Phi \mid-r^{-1} \partial_{r^{*}}^{2}(r \Phi)\right\rangle \\
& =\left\|r^{-1} \partial_{r^{*}}(r \Phi)\right\|^{2}
\end{aligned}
$$

The second inequality above follows from $V_{1}-V_{2} \widehat{D}^{A} \widehat{D}_{A}$ being positive definite on fields in $L^{2}\left(S^{2}\right)>1$, the integration by parts in the last line is trivial for the compactly supported $\Phi_{(a)}$ and, in view of $(227)-(228)$, holds for $\Phi_{(b)}$. The 
third term in (229) is similarly bounded by $\mathcal{E}\left[\mathbf{J}^{2} \Phi\right]$. To treat the second term on the right hand side of (229) we proceed as in [8], by taking the $r^{*}$ derivative of $\mathcal{A}^{\Lambda}(r \Phi)$ (see (67))

$$
\partial_{r^{*}}^{3}(r \Phi)=-\partial_{r^{*}} \mathcal{A}^{\Lambda}(r \Phi)+r \Phi \partial_{r^{*}} V_{1}+V_{1} \partial_{r^{*}}(r \Phi)-\left(\partial_{r^{*}} V_{2}\right) \mathbf{J}^{2}(r \Phi)-V_{2} \mathbf{J}^{2} \partial_{r^{*}}(r \Phi)
$$

and using the facts that $\Phi^{\prime}=r^{-1} \mathcal{A}^{\Lambda}(r \Phi)$ is also a solution of the 4DRWE and that the $V_{i}$ 's and their $r^{*}$ derivatives are bounded in region II: $\left|V_{i}\right| \leq V_{i}^{\max },\left|\partial_{r^{*}} V_{i}\right| \leq V_{i, r^{*}}^{\max }$ :

$$
\begin{array}{r}
\left\|r^{-1} \partial_{r^{*}}^{3}(r \Phi)\right\| \leq\left\|r^{-1} \partial_{r^{*}} \mathcal{A}^{\Lambda}(r \Phi)\right\|+\|\Phi\| V_{1, r^{*}}^{\max }+V_{1}^{\max }\left\|r^{-1} \partial_{r^{*}}(r \Phi)\right\|+V_{2, r^{*}}^{\max }\left\|r^{-1} \mathbf{J}^{2}(r \Phi)\right\|+V_{2}^{\max }\left\|r^{-1} \mathbf{J}^{2} \partial_{r^{*}}(r \Phi)\right\| \\
\leq \sqrt{\mathcal{E}\left[r^{-1} \mathcal{A}^{\Lambda}(r \Phi)\right]}+\sqrt{\mathcal{E}[\tilde{\Phi}]} V_{1, r^{*}}^{\max }+V_{1}^{\max } \sqrt{\mathcal{E}[\Phi]}+V_{2, r^{*}}^{\max } \sqrt{\mathcal{E}\left[r^{-1} \mathbf{J}^{2}(r \tilde{\Phi})\right]}+V_{2}^{\max } \sqrt{\mathcal{E}\left[r^{-1} \mathbf{J}^{2}(r \Phi)\right]}(232)
\end{array}
$$

The finiteness of the energy integrals above for $\Phi=\Phi_{(b)}$ can easily be checked. We conclude that there is a constant $K^{\prime}$ such that, in region II,

$$
K^{\prime} \geq\left|\partial_{r^{*}}(r \Phi)\right| \geq f|\Phi|+r\left|\partial_{r^{*}} \Phi\right|
$$

and, since $f$ and $\Phi$ are bounded in this region,

$$
\left|\partial_{r *} \Phi\right| \leq \frac{K^{\prime \prime}}{r}
$$

for some constant $K^{\prime \prime}$. Thus, every term in (215) is bounded by a constant times $r^{-4}$.

\section{DISCUSSION}

\section{A. Evolution of perturbations}

The large $t$ decay of $\phi_{(\ell, m)}^{ \pm}[25,39]$, together with equations

$$
G_{-}=-\frac{6 M}{r^{7}} \sqrt{\frac{4 \pi}{3}} \sum_{m=1}^{3} j^{(m)} S_{(1, m)}-\frac{3 M}{r^{5}} \sum_{(\ell \geq 2, m)} \frac{(\ell+2) !}{(\ell-2) !} \frac{\phi_{(\ell, m)}^{-}}{r} S_{(\ell, m)}
$$

and

$$
G_{+}=-\frac{2 M \dot{M}}{r^{5}}+\frac{M}{2 r^{4}} \sum_{\ell \geq 2} \frac{(\ell+2) !}{(\ell-2) !}\left[f \partial_{r}+Z_{\ell}\right] \phi_{(\ell, m)}^{+} S_{(\ell, m)},
$$

indicate that, at large $t$,

$$
G_{-} \sim-\frac{6 M}{r^{7}} \sqrt{\frac{4 \pi}{3}} \sum_{m=1}^{3} j^{(m)} S_{(1, m)}, \quad G_{+} \sim-\frac{2 M \dot{M}}{r^{5}}
$$

which, in view of the bijection (9), corresponds to a linearized Kerr (Kerr de Sitter) black hole around the Schwarzschild (SdS) background. The picture that emerges from these considerations is that, for a generic perturbation, the black hole ends up settling into a slowly rotating Kerr (Kerr dS) black hole. To make statements like these more precise, all we need is quantitative information on the decay of solutions of the $4 \mathrm{DRW}$ equation. This is so because $r^{5} G_{-} i s$ a solution of this equation, and $G_{+}$can be written entirely in terms of solutions of the 4 DRW equation (see equation (215)). Alternatively, in view of Theorem 4, there is a bijection between solutions of the LEE and a set containing the constants $\dot{M}, j^{(m)}$ and two fields $\Phi_{ \pm}$that obey the 4DRW equation. The perturbed metric is linearized Kerr (Kerr de Sitter) if $\Phi_{ \pm}=0$, which is the limit approached at large $t$. Quantitative results for the decay in time of solutions of the 4DRW equation can be found in [1], [11] and more recently in [7] (in this last reference, as statements on a symmetric tensor field made out of two scalar fields obeying (4DRWE)). 


\section{B. Reduction of the LEE to the $4 \mathrm{DRW}$ equation}

A natural question that arises from our results is: can we forget altogether metric perturbations and restrict ourselves to the study of the 4DRW equation? The answer to this question is in the affirmative (Theorem 4 and equations (104) and (215)), although some technical issues should be mentioned if one intends to study perturbations using only the $G_{ \pm}$or similar perturbed curvature fields. One is related to the possibility of evolving perturbations from initial $G_{ \pm}$data. Since $r^{5} G_{-}$obeys the 4 DRW equation, initial data (e.g., $\partial_{t} G_{-}$and $G_{-}$restricted to a $t=$ constant hypersurface) gives $G_{-}$and, by expanding in spherical harmonics, the metric perturbation in the RW gauge uniquely related to it. This does not happen, however, with $G_{+}$in the even sector. Since the operator $f \partial_{r}+Z_{\ell}$ in $(204)$ has a non trivial kernel, and $G_{+}$does not satisfy a wave equation (which would allow us to recover the lost information by proceeding as in Section IVE), the set of $\partial_{t} \phi_{(\ell, m)}^{+}$and $\phi_{(\ell, m)}^{+}$at $t=t_{o}$ cannot be obtained from $\partial_{t} G_{+}$and $G_{+}$ at $t=t_{o}$. The reason why $G_{+}$does not obey a wave equation is, of course, that is made out of the perturbation of curvature scalars involving both the Riemann tensor and its covariant derivative; the possibility of constructing a gauge invariant curvature scalar field for the even sector that does not use derivatives of the Riemann tensor was ruled out in $[9]$.

We should mention, however, two alternatives to the use of $G_{+}$(or similar fields involving CSs). One is using the potentials $\Phi_{ \pm}$in Theorem 4: perturbations are entirely characterized by $\dot{M}, j^{(m)}$ (stationary perturbations) and the fields $\Phi_{ \pm}$obeying the 4DRW equation (dynamical perturbations). This shows that we can do without the Zerilli equation and that the LEE does reduce to the 4DRW equation. The other possibility to avoid scalar fields involving higher metric derivatives (such as $\dot{X}$ in $G_{+}$, equation (202)), is constructing gauge invariant combinations made out of perturbed curvature scalars and the metric perturbation. We close this section by exhibiting an example of such a construction: if we calculate the (gauge dependent) scalar field $\dot{Q}_{+}($see $(7))$ in the RW gauge, we find that

$$
\dot{Q}_{+}^{(R W)}=\frac{2 M \dot{M}}{r^{6}}-\frac{6 M^{2}}{r^{5}} \sum_{(\ell \geq 2, m)} \frac{\mathcal{D}_{\ell}^{-} \phi_{(\ell, m)}^{+}}{r} S_{(\ell, m)},
$$

thus $r^{5} \dot{Q}_{+,>1}^{(R W)}$ also satisfies the 4DRW equation! We might think of using $\dot{Q}_{+}$to measure the strength of even perturbations, but this field is gauge dependent due to the fact that $Q_{+} \neq 0$ for the background Schwarzschild or $\mathrm{S}(\mathrm{A}) \mathrm{dS}$ black hole. Under the gauge transformation (70),

$$
\dot{Q}_{+} \rightarrow \dot{Q}_{+}{ }^{\prime}=\dot{Q}_{+}+\zeta^{r} \partial_{r} Q_{+}=\dot{Q}_{+}-\zeta^{r} \frac{6 M^{2}}{r^{7}} .
$$

This suggests searching for a gauge invariant field $H_{+}$that agrees with $\dot{Q}_{+}$in the RW gauge. If we compare (237) with equation (126), we find the following solution:

$$
H_{+}=\frac{6 M^{2}}{r^{7}} \widetilde{D}^{a} r\left(q_{a}-r^{2} \widetilde{D}_{a} G\right)+\dot{Q}_{+}
$$

Since this gauge invariant field reduces in the $R W$ gauge to $\dot{Q}_{+}^{(R W)}$, and the $\phi_{(\ell, m)}^{+}$are gauge invariant,

$$
H_{+}=\frac{2 M \dot{M}}{r^{6}}-\frac{6 M^{2}}{r^{5}} \sum_{(\ell \geq 2, m)} \frac{\mathcal{D}^{-} \phi_{(\ell, m)}^{+}}{r} S_{(\ell, m)} .
$$

Note that the dynamical $\ell \geq 2$ piece of $r^{5} H_{+}$satisfies the 4 DRW equation, which gives $H_{+}$an advantage over $G_{+}$: it is possible to obtain $H_{+}$from the initial datum $\left.\left(r^{5} H_{+}, r^{5} \partial_{t} H_{+}\right)\right|_{t_{o}}$. Once this is done, the corresponding metric perturbation in the RW gauge can be obtained by expanding $H_{+}$in spherical harmonics, which gives us $\dot{M}$ and the $\mathcal{D}_{\ell}^{-} \phi_{(\ell, m)}^{+}$and then applying Lemma 7 to recover the Zerilli fields $\phi_{(\ell, m)}^{+}$. Although the $H_{+}$looks more geometrical than the potentials $\Phi_{ \pm}$in Theorem 4 , we have not found an obvious interpretation for this field.

\section{ACKNOWLEDGMENTS}

I thank Sergio Dain for suggesting writing a detailed version of the proof of nonmodal linear stability in [9], Andrés Anabalón for suggesting considering extending this proof to the case of nonzero cosmological constant, Gustav Holzegel for pointing out an error in the proof of Theorem 6 in a previous version of this manuscript and Reinaldo Gleiser 
and Martin Reiris for useful discussions. This work was partially funded by grants PIP 11220080102479 (ConicetArgentina) and Secyt-UNC 05/B498 (Universidad Nacional de Córdoba).

[1] P. Blue and A. Soffer, "The Wave equation on the Schwarzschild metric. 2. Local decay for the spin 2 Regge-Wheeler equation," J. Math. Phys. 46, 012502 (2005) doi:10.1063/1.1824211 [gr-qc/0310066].

[2] L. T. Buchman and O. C. A. Sarbach, "Improved outer boundary conditions for Einstein's field equations," Class. Quant. Grav. 24, S307 (2007) doi:10.1088/0264-9381/24/12/S20 [gr-qc/0703129].

[3] S. Chandrasekhar, "On algebraically special perturbations of black holes", Proc. R. Soc. Lond. A 392 (1984) 1.

[4] S. Chandrasekhar, "The Mathematical Theory of Black Holes", Oxford University Press, second edition, 1992.

[5] E. Chaverra, N. Ortiz and O. Sarbach, "Linear perturbations of self-gravitating spherically symmetric configurations," Phys. Rev. D 87, no. 4, 044015 (2013) doi:10.1103/PhysRevD.87.044015 [arXiv:1209.3731 [gr-qc]].

[6] Christodoulou, D. and Klainerman, S. The global nonlinear stability of the Minkowski space. Princeton: Princeton University Press (1993).

[7] M. Dafermos, G. Holzegel and I. Rodnianski, "The linear stability of the Schwarzschild solution to gravitational perturbations," arXiv:1601.06467 [gr-qc].

[8] S. Dain and G. Dotti, "The wave equation on the extreme Reissner-Nordstróm black hole," Class. Quant. Grav. 30, 055011 (2013) doi:10.1088/0264-9381/30/5/055011 [arXiv:1209.0213 [gr-qc]].

[9] G. Dotti, "Non-modal linear stability of the Schwarzschild black hole," Phys. Rev. Lett. 112 (2014) 191101 [arXiv:1307.3340 $[\mathrm{gr}-\mathrm{qc}]]$.

[10] G. Dotti and R. J. Gleiser, "The initial value problem for linearized gravitational perturbations of the Schwarzchild naked singularity," Class. Quant. Grav. 26, 215002 (2009) doi:10.1088/0264-9381/26/21/215002 [arXiv:0809.3615 [gr-qc]].

[11] J. Friedman and M. Morris, "Schwarzschild perturbations die in time," J. Math. Phys. 41 (2000) 7529.

[12] U. H. Gerlach and U. K. Sengupta, "Gauge Invariant Perturbations On Most General Spherically Symmetric Space-times," Phys. Rev. D 19, 2268 (1979).

[13] A. Ishibashi and H. Kodama, "Stability of higher dimensional Schwarzschild black holes," Prog. Theor. Phys. 110, 901 (2003) doi:10.1143/PTP.110.901 [hep-th/0305185].

[14] H. Kodama and A. Ishibashi, "A Master equation for gravitational perturbations of maximally symmetric black holes in higher dimensions," Prog. Theor. Phys. 110, 701 (2003) doi:10.1143/PTP.110.701 [hep-th/0305147].

[15] G. W. Gibbons and S. W. Hawking, "Cosmological Event Horizons, Thermodynamics, and Particle Creation," Phys. Rev. D 15, 2738 (1977). doi:10.1103/PhysRevD.15.2738

[16] R. J. Gleiser and G. Dotti, "Instability of the negative mass Schwarzschild naked singularity," Class. Quant. Grav. 23, 5063 (2006) doi:10.1088/0264-9381/23/15/021 [gr-qc/0604021].

[17] J. Guven and D. Núñez, "Schwarzschild-de Sitter space and its perturbations", Phys. Rev. D 42 (1990) 2557.

[18] A. Higuchi, "Symmetric Tensor Spherical Harmonics on the $N$ Sphere and Their Application to the De Sitter Group SO $(N, 1)$," J. Math. Phys. 28 (1987) 1553 [Erratum-ibid. 43 (2002) 6385].

[19] A. Ishibashi and R. M. Wald, "Dynamics in nonglobally hyperbolic static space-times. 2. General analysis of prescriptions for dynamics," Class. Quant. Grav. 20, 3815 (2003) doi:10.1088/0264-9381/20/16/318 [gr-qc/0305012].

[20] A. Ishibashi and R. M. Wald, "Dynamics in nonglobally hyperbolic static space-times. 3. Anti-de Sitter space-time," Class. Quant. Grav. 21 (2004) 2981 [hep-th/0402184].

[21] B. S. Kay and R. M. Wald, "Linear Stability Of Schwarzschild Under Perturbations Which Are Nonvanishing On The Bifurcation Two Sphere," Class. Quant. Grav. 4, 893 (1987).

[22] K. Lake and R. C. Roeder, "Effects of a nonvanishing cosmological constant on the spherically symmetric vacuum manifold", Phys. Rev. D 15, 3513 (1976).

[23] V. Moncrief, "Gravitational perturbations of spherically symmetric systems. I. The exterior problem.," Annals Phys. 88, 323 (1974).

[24] V. Pravda, A. Pravdova, A. Coley and R. Milson, "All space-times with vanishing curvature invariants," Class. Quant. Grav. 19, 6213 (2002) doi:10.1088/0264-9381/19/23/318 [gr-qc/0209024].

[25] R. H. Price, "Nonspherical perturbations of relativistic gravitational collapse. 1. Scalar and gravitational perturbations," Phys. Rev. D 5, 2419 (1972). doi:10.1103/PhysRevD.5.2419

[26] T. Regge and J. A. Wheeler, "Stability of a Schwarzschild singularity," Phys. Rev. 108, 1063 (1957).

[27] O. Sarbach and M. Tiglio, "Gauge invariant perturbations of Schwarzschild black holes in horizon penetrating coordinates," Phys. Rev. D 64, 084016 (2001) [gr-qc/0104061].

[28] K. Schleich and D. M. Witt, "A simple proof of Birkhoff's theorem for cosmological constant," J. Math. Phys. 51, 112502 (2010) doi:10.1063/1.3503447 [arXiv:0908.4110 [gr-qc]].

[29] R. Wald, "Note on the stability of the Schwarzschild metric", J. Math. Phys. 20, 1056 (1979), Erratum J. Math. Phys. 21, 218 (1980).

[30] Schmid, Peter J. "Nonmodal stability theory." Annu. Rev. Fluid Mech. 39 (2007): 129.

[31] R. M. Wald, "Dynamics In Nonglobally Hyperbolic, Static Space-times," J. Math. Phys. 21, 2802 (1980). doi:10.1063/1.524403 
[32] F. J. Zerilli, "Effective potential for even parity Regge-Wheeler gravitational perturbation equations," Phys. Rev. Lett. 24, 737 (1970).

[33] B. Araneda and G. Dotti, work in progress.

[34] E. Berti, V. Cardoso and A. O. Starinets, "Quasinormal modes of black holes and black branes," Class. Quant. Grav. 26, 163001 (2009) doi:10.1088/0264-9381/26/16/163001 [arXiv:0905.2975 [gr-qc]].

[35] V. Cardoso and J. P. S. Lemos, "Quasinormal modes of Schwarzschild anti-de Sitter black holes: Electromagnetic and gravitational perturbations," Phys. Rev. D 64, 084017 (2001) doi:10.1103/PhysRevD.64.084017 [gr-qc/0105103].

[36] B. Araneda and G. Dotti, "Petrov type of linearly perturbed type D spacetimes," Class. Quant. Grav. 32, no. 19, 195013 (2015) doi:10.1088/0264-9381/32/19/195013 [arXiv:1502.07153 [gr-qc]].

[37] T. Andrade, W. R. Kelly, D. Marolf and J. E. Santos, "On the stability of gravity with Dirichlet walls," Class. Quant. Grav. 32, no. 23, 235006 (2015) doi:10.1088/0264-9381/32/23/235006 [arXiv:1504.07580 [gr-qc]]

[38] I. Bakas, "Energy-momentum/Cotton tensor duality for AdS(4) black holes," JHEP 0901, 003 (2009) doi:10.1088/11266708/2009/01/003 [arXiv:0809.4852 [hep-th]].

[39] P. R. Brady, C. M. Chambers, W. Krivan and P. Laguna, "Telling tails in the presence of a cosmological constant," Phys. Rev. D 55, 7538 (1997) doi:10.1103/PhysRevD.55.7538 [gr-qc/9611056]. 